\title{
Polymer Electrolyte Water Electrolysis: Correlating Porous Transport Layer Structural Properties and Performance: Part I. Tomographic Analysis of Morphology and Topology
}

\author{
Tobias Schuler, ${ }^{1}$ Ruben De Bruycker, ${ }^{2}$ Thomas J. Schmidt, ${ }^{1,3, *}$ and Felix N. Büchi ${ }^{1, z}$ \\ ${ }^{I}$ Electrochemistry Laboratory, Paul Scherrer Institut, CH-5232 Villigen PSI, Switzerland \\ ${ }^{2}$ NV Bekaert SA, 8550 Zwevegem, Belgium \\ ${ }^{3}$ Laboratory of Physical Chemistry, ETH Zürich, CH-8093 Zürich, Switzerland
}

\begin{abstract}
In the first paper of this series, the bulk, surface and transport properties of porous transport layer materials (PTL) for polymer electrolyte water electrolysis cells (PEWE) are characterized. A systematic PTL matrix of Ti fiber materials with 3 fiber diameters and 2 nominal porosities as well as a state of the art sintered powder material are investigated to get a better understanding of the governing parameters in electrolysis cells. X-ray tomographic microscopy analysis of ex situ PTL structures and post operando membrane electrode assemblies is performed. On the tomographic structures, bulk (porosity, pore/solid size distributions, fiber orientation), mass transport (diffusivity, permeability, conductivities) and surface parameters (roughness, membrane deformation, PTL surface area and interfacial contact area) are determined. The second paper of this series will correlate the structural parameters with in-depth electrochemical analysis. The new insights into the effect of PTL properties on PEWE performance allow to isolate governing key parameters. From know-how obtained on the fiber based materials fundamental design guidelines for optimization of PTL structures are deduced.

(C) The Author(s) 2019. Published by ECS. This is an open access article distributed under the terms of the Creative Commons Attribution Non-Commercial No Derivatives 4.0 License (CC BY-NC-ND, http://creativecommons.org/licenses/by-nc-nd/4.0/), which permits non-commercial reuse, distribution, and reproduction in any medium, provided the original work is not changed in any way and is properly cited. For permission for commercial reuse, please email: oa@electrochem.org. [DOI: 10.1149/2.0561904jes]
\end{abstract}

(cc) BY-NC-ND

Manuscript submitted December 13, 2018; revised manuscript received February 8, 2019. Published March 2, 2019.

To limit the greenhouse gases based global temperature increase by 2050 to $2^{\circ} \mathrm{C}$, average $\mathrm{CO}_{2}$ emissions per unit of final energy supplied of $90 \mathrm{~kg} \mathrm{GJ}^{-1}$ has to be reduced to $19.5-37.0 \mathrm{~kg} \mathrm{GJ}^{-1}$. ${ }^{2}$ Therefore a transition to net zero $\mathrm{CO}_{2}$ renewable energy carriers is inescapable. However the fluctuating nature of many renewable energy sources is a significant challenge for their integration into the energy systems. Diurnal and seasonal misfit of production and consumption needs to be balanced by storage.

Hydrogen is a suited storage vector. Polymer electrolyte water electrolysis (PEWE) provides the required properties of dynamic operation paired with high current densities and high purity hydrogen production. ${ }^{3}$ Today's relatively high capital and operational cost need to be reduced to make the technology competitive for energy storage applications. ${ }^{4}$ Operational cost is reduced by improving cell efficiency at given current density (if power cost is considered an external variable). PEWE current collectors, termed porous transport layers (PTL) at the anode side are commonly based on titanium due to its electrochemical stability at the high potentials of the oxygen evolution reaction (OER). It is of interest to design PTLs to minimize electrochemical losses related to their structure. Therefore PTL design rules are required guiding the balance of cell efficiency and possibly PTL costs. The governing role of PTLs in the cell is given due to their electric and thermal connection of flow field and catalyst coated membranes (CCM) while providing optimum transport for water and product gases. The impact of the transport layer structure on mass transport overpotential, in the past mainly related to two phase flow in the porous media, ${ }^{5-7}$ appears to play a crucial role in PEWE. Mostly screening processes of a wide range of different porous transport layers such as Ti sheets, ${ }^{5,8}$ foams, ${ }^{9}$ grids ${ }^{10}$ and sintered structures ${ }^{5,11-13}$ have been conducted to characterize their suitability and performance. Generally, bulk characteristics such as pore and particle size distributions and transport parameters were related to electrochemical performance data in various studies.

Gregoriev et al. ${ }^{12}$ focused on the optimization of PTLs based on spherical sintered Ti particles with a porosity range of $28 \%-40 \%$. The transport layers were characterized electrochemically, but correlation

\footnotetext{
*Electrochemical Society Member
}

${ }^{\mathrm{z}}$ E-mail: felix.buechi@psi.ch with the structure was only made based on general geometric concepts. The authors pointed out that morphological properties impact the overall overpotential and suggested an ideal pore diameter in the range of $12-13 \mu \mathrm{m}$ for spheres in the range of 50-75 $\mu \mathrm{m}$. Ito et al. ${ }^{14,15}$ suggested preferential pore diameters in the range of $10 \mu \mathrm{m}$ based on data from experiments with Ti fiber meshes with $46 \%-75 \%$ porosity.

Besides electrochemical characterization, optical imaging using transparent cell designs was conducted to investigate flow regimes and mass transport. Dedigema et al. ${ }^{16,17}$ showed that the transition of two-phase flow in the flow channel from bubbly to slug flow improves fluidic transport. Arbabi et al. ${ }^{18,19}$ characterized oxygen bubble growth based on microfluidic chips mimicking the bulk properties of a PTL. The studies showed limiting throat sizes dominate gas pathways.

Imaging techniques were used to relate PTL bulk properties with fluid transport. Studies made with in-plane and through-plane neutron imaging $^{20,21}$ and X-ray radiography ${ }^{22,23}$ revealed constant gas/water fractions in the PTL and selective gas pathways at the interface of PTL and flow-field. An ex situ X-ray tomographic micrsoscopy (XTM) analysis of PTL morphologies was conducted by Zielke et al. ${ }^{24}$ They proposed an universal empirical correlation between conductivity and permeability of the PTL's dry structure.

Another structural impact factor with possible influence on the transport overpotential is the PTL and catalyst layer (CL) interface. Insight into the influence of the PTL/CL interface was reported by Mo et al. ${ }^{25}$ Using a transparent flow field and a high speed camera they observed oxygen bubble evolution exclusively at the edges of the perforated Ti sheets and CL interface, not in the middle of pores, emphasizing the importance of the PTL/CL interface structure. This result highlights the importance of the PTL/CL interface structure, implying that optimizing the PTL interface results in a performance increase.

Therefore, surface modified PTLs have been investigated. Hwang et al. ${ }^{26,27}$ used $\mathrm{Ti}$ powder on Ti felt to create a microporous layer (similar to those used in polymer electrolyte fuel cells). With tests based on unitized reversible fuel cells they investigated different contents of PTFE modifying the hydrophobic character of the PTL. However, the performance was not noticeably affected by the different PTFE contents. Lettenmeier et al. ${ }^{11,28}$ used vacuum plasma spray (VPS) coatings for manufacturing graded PTLs. A decrease in ohmic 

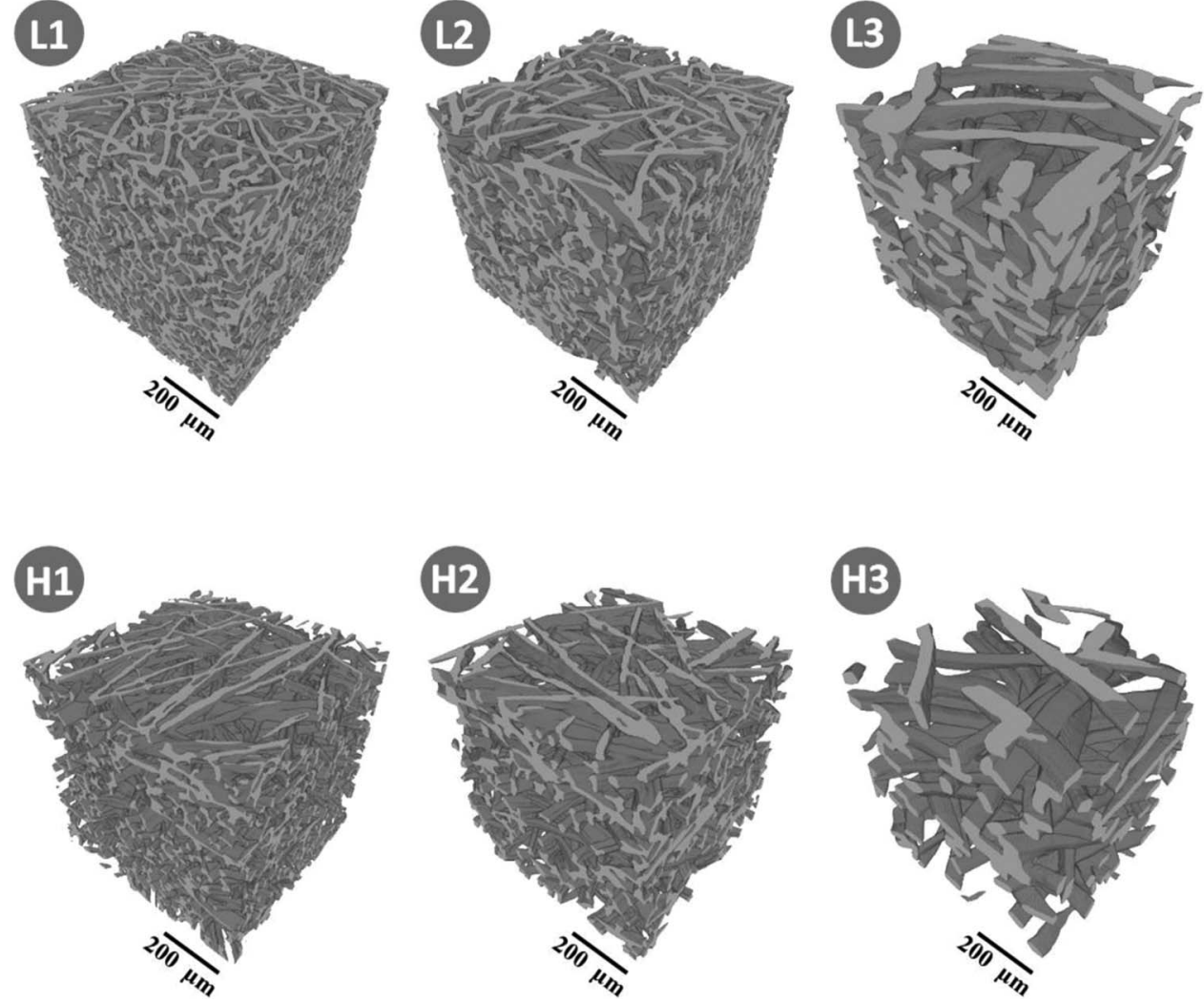

Figure 1. 3D surface renderings based on XTM data of the three fiber types 1, 2, 3 shown for the two nominal porosities of 56\% (L) and 76\% (H). The Ti solid phase is represented in gray and void phase in transparent, respectively.

resistance and improved performance was observed for structures with additional VPS layer on top of a commercially available Ti-powder sintered supports. No change in the kinetic region $\left(<0.1 \mathrm{~A} / \mathrm{cm}^{2}\right)$ was observed when comparing original and VPS-modified transport layers. ${ }^{11}$

The studies reported in literature addressed the impact of bulk and surface properties separately. Only very limited design rules for bulk and even less for interface PTL structures were established due to missing correlation of ex situ and operando results, because no detailed overpotential analysis was performed or a limited/unstructured sample matrix was used, which did not allow for deducing general concepts.

Sintered titanium fiber based porous transport layers are a wellstablished class of materials in PEWE. This study aims at providing the missing link between the surface and bulk structures of PEWEs and the three main losses for PEWE operation, induced by charge transport, mass transport and electrochemical kinetics. No well-defined correlation between the structural properties of the transport layer materials and the influence on performance in PEWE has to date been established. Therefore a series of Ti fiber based PTLs is investigated. The materials based on three different nominal Ti fiber diameters $(14,22$ and $50 \mu \mathrm{m})$ and two different porosities $(56 \%$ and $76 \%$ ) leading to a sample matrix with systematically different pore and throat size distributions, as well as transport and surface properties.

In this first part of the investigation, transport layer morphology representing the bulk properties of a material, as well as topology capturing the surface properties of a material, are characterized based on X-ray tomographic microscopy. Morphological properties of the PTL bulk phase such as porosity, tortuosity and the size distributions of pores and particles (PSDs) are determined. In addition to the conventional continuous PSD, simulated mercury intrusion porosimetry ${ }^{29}$ and the in-plane particle orientation are analyzed. To address the topological properties of the materials, the surface roughness is determined and a new methodology for PTL/CL interface characterization is de- veloped based on a combination of ex situ and structural post operando catalyst layer analysis using XTM imaging. This approach enables the quantification of interfacial contact area (ICA) at the interface of PTL and $\mathrm{CL}$ which will be identified as a governing property for PEWE performance.

The second paper of this series will focus on the electrochemical analysis of PEWE cells based on the same PTL material matrix. Electrochemical performance is systematically correlated to bulk and surface structures by a detailed electrochemical loss break down. Based on the obtained quintessence for the fiber based materials, design guidelines can be formulated for optimum PEWE performance of all material classes.

\section{Experimental}

Materials.-A systematic PTL matrix based on three different nominal fiber diameters $(14,22,50 \mu \mathrm{m}$ labeled as diameters $1-3$, respectively) and two nominal porosities of $56 \%$ (labeled $\mathrm{L}$ for low) and $76 \%$ ( $\mathrm{H}$ for high), as shown in Figure 1, was manufactured by NV Bekaert SA (Belgium) for this study.

The sintered titanium fibers are produced by spreading titanium fibers in a uniform non-woven. Subsequent heat-treatment results in inter-metallic diffusion bonding of the individual fibers. The raw material of the fibers is grade 1 Titanium. Samples of $20 \mathrm{~mm} \times 20 \mathrm{~mm}$ were cut by electrical discharge machining.

All materials had a nominal thickness of $1 \mathrm{~mm}$. A thickness deviation of $+/-0.2 \%-6.4 \%$ was measured with a thickness gauge MarCator 1075R (Mahr GmbH, Germany). This is significantly less as compared to commercially available Ti powder sintered materials e.g. SIKA Ti-powder sintered materials (GKN Sinter Metals Filters $\mathrm{GmbH}$, Germany) originally developed for filtration, showing thickness deviations of -2 to $25 \% .^{5}$ In this work, the commercially available, material SIKA T10 was used as reference PTL for comparison. 
X-ray tomographic microscopy.-The significant difference of X-ray mass attenuation coefficient between Ti and air of $1.95 \mathrm{~cm}^{2} / \mathrm{g}$ (contrast@ $90 \mathrm{keV}$, monochromatic) ${ }^{30}$ makes XTM a well suited tool for structural analysis. Surface and bulk properties were derived from dry structure imaging using a CT-scanner phoenix nanotom $\mathrm{m}$ (General Electric, Germany). Samples with dimensions of $20 \times 2.0 \times$ $1.0 \mathrm{~mm}^{3}$ were mounted vertically for imaging. A $0.25 \mathrm{~mm}$ copper filter was used to suppress beam hardening effects. Acquisition parameters were set to $60 \mathrm{kV}$ and $310 \mu \mathrm{A}$. A voxel cube edge length of $2 \mu \mathrm{m}$ was chosen for all PTL samples resulting in a minimum of 5 to 6 voxels/feature size. 1500 projections over $360^{\circ}$ were acquired with an exposure time of $1500 \mathrm{~ms}$, resulting in an acquisition time per sample of approximately $2 \mathrm{~h}$. To characterize the membrane deformation on the surface of the PTL, $20 \times 20 \mathrm{~mm}^{2}$ electrochemically operated CCM/PTL sandwiches were imaged directly after cell disassembling. To ensure a reliable analysis of plastic membrane deformation, exclusively CCM/PTL sandwiches adhering to each other after disassembly were analyzed. The high attenuation coefficients of the precious metal catalysts in the anodic and cathodic electrodes made catalyst layer deformation easily observable. For this post operando imaging, local tomography was conducted with a voxel size of $3 \mu \mathrm{m}$ resulting in 3 to 4 voxels for the catalyst layer thickness. Prior to imaging, the samples were hydrated with deionized water and sealed with parafilm to prevent membrane shrinking during image acquisition.

Image processing.-All image processing steps were conducted in the open source software ImageJ, unless stated otherwise. The raw data was corrected for misalignment in all three dimensions with reference to the PTL surfaces. The corresponding orientation correction is essential for topological analysis. The significant contrast between void and solid required solely an anisotropic diffusion filter to intensify sharp edge enhancement. A manually guided threshold, suggested by the Otsu method, ${ }^{31}$ was subsequently performed.

Isolation of deformed catalyst layer was conducted by low frequency Hessian filtering before manual thresholding. A 3D based clearing of clusters of less than 30'000 voxels was performed in Matlab to remove outliers in the void volume. Finally a $3 \mathrm{D}$ erosion/dilation step was applied.

Region of interest.-The mechanical cutting of PTL materials samples for imaging leads to edge effects by sample melting. Therefore, only the unharmed matrix in the sample bulk was used for evaluation. For bulk characterization, the outermost $50 \mu \mathrm{m}$ of the PTL surfaces were excluded for the region of interest (ROI) of $2000 \times$ $1000 \times 900 \mu^{3}$. The ROI of the PTL surface analysis covered a geometric area of $2000 \times 1000 \mu \mathrm{m}^{2}$. The sizes of the ROIs for post operando analysis (all $>1.8 \mathrm{~mm}^{2}$ ) are reported in Table II.

$\boldsymbol{R} \boldsymbol{E} \boldsymbol{V}$.- The representative elementary volume (REV) defines the smallest volume element which adequately possesses the bulk properties of the material according to statistic criteria. The PTL porosity was defined as the bulk property of interest for the REV analysis. A maximum standard deviation of $3 \%$ between sample volumes and porosity was the chosen criterion in this study.

Bulk properties.-To quantify the global pore and particle size distribution (PSD) of the bulk phase a 3D maximum sphere fitting was conducted with the ImageJ plug-in local thickness. ${ }^{32}$ The minimum throat size of pores called bottlenecks can be identified by comparing continuous PSD with computed mercury intrusion porosimetry PSD (MIP-PSD). ${ }^{29}$ For MIP-PSD, artificial spheres with different radii are introduced into the pore space from a defined PTL surface. The spheres diffuse in the through-plane direction. Local pores acting as bottlenecks limit selectively the access to further PTL pore volume.

The fiber-based PTL materials have an anisotropic structure with fibers approximately stacked in layers, see Figure 1. Therefore 2-D fiber orientations were computed for every fiber voxel based on its structural tensor. ${ }^{33}$
Transport properties.-Permeability $\kappa$, bulk diffusivity $D_{e f f}$, tortuosity $\tau$, effective electrical and thermal conductivities $\sigma$ were computed at $50^{\circ} \mathrm{C}$ in all space directions using the software GeoDict (Math2Market, Germany). The global boundary conditions were identical for all computed transport parameters: Dirichlet boundary conditions in the main computed direction were selected over periodic boundary conditions to avoid artificial closing of open pores at the surface. Periodic boundary conditions were set for lateral directions. An accuracy of 0.001 was requested as default stopping criteria. The thermal and electric material conductivities were set to $0.6 \mathrm{~W} / \mathrm{mK}$ $\left(\mathrm{H}_{2} \mathrm{O}\right)$ and $21.9 \mathrm{~W} / \mathrm{mK}$ (Ti) for thermal conductivity and to $2.38 \cdot 10^{6}$ $\mathrm{S} / \mathrm{m}$ (Ti) for electrical conductivity.

For the determination of the effective diffusivity and tortuosity in the void space, molecular diffusion was chosen. Knudsen diffusion can be ignored at with pore sizes of $d_{p}=50 \mu \mathrm{m}$ at $\mathrm{p}=1$ bar and $\mathrm{T}=50^{\circ} \mathrm{C}$, with a Knudsen number of $\mathrm{K}_{\mathrm{n}} \sim 0.001$, which is considerably smaller than the usually applied criteria of $K_{n}>0.1$. The water permeability was determined by the linear flow solution method in combination with a 20 voxel thick inflow region.

Post operando analysis.-Commercial catalyst coated membranes (CCMs Greenerity E400, Greenerity, DE) were used for electrochemical characterization. Nafion 117CS featuring a dry thickness of $175 \mu \mathrm{m}$ is used as membrane. An in-house designed differential cell with an active area of $4 \mathrm{~cm}^{2}$ and parallel flow fields (5 channels $20 \times 2 \times 1 \mathrm{~mm}^{3}, 2 \mathrm{~mm}$ ribs) was used. ${ }^{34}$ A contact pressure of $2.5 \mathrm{MPa}$ is applied by a spring configuration providing a constant contact pressure independent of PTL thickness deviations. A symmetric CCM/PTL configuration with PTL on both sides was operated for $60-70 \mathrm{~h}$ at $50^{\circ} \mathrm{C}$ and $10 \mathrm{bar}$ before post operando analysis.

\section{Definition and Determination of Surface Roughness}

The surface roughness $R$, is utilized in different fields to quantify the topological properties of a material. Generally, the parameters describing $R$ can be divided into four categories (amplitude, spatial, functional and hybrid). ${ }^{35,36}$ This study focuses on the amplitude based parameter given by profile height $t_{i j}$ and hybrid based parameter related to surface defined as the surface area (SA). Both parameters appear to be of electrochemical relevance due to the interaction of the PTL's surface with the catalyst coated membrane (CCM) resulting in membrane deformation and a corrugated PTL/CL interface.

Amplitude based surface roughness. - The total profile height $t_{i j}$ at probing position $i$ and $j$ is defined by the length of a normal vector to a reference plane which is parallel to the surface of the analyzed material. A material surface is characterized by a total number of surface probing points of $\mathrm{M} \times \mathrm{N}$.

Based on the obtained profile height matrix, the mean surface roughness $R_{m}$ can be calculated according to Eq. $1 . R_{m}$ represents the average total profile height of the material's surface. A high value of $R_{m}$ indicates a rough surface profile whereas a perfectly flat surface would result in a $R_{m}$ of $0 \mu \mathrm{m}$. In this study, $R_{m}^{P T L}$ is used to characterize the dry structure surface of the PTL according to Eq. 1.

$$
R_{m}^{P T L}=\frac{1}{M N} \sum_{i=0}^{M-1} \sum_{j=0}^{N-1} t_{i j}^{P T L}
$$

The same concept can be transferred to membrane deformation. The irreversible plastic deformation of the membrane is defined by the deformation height $t_{i j}^{\text {mem }}$. The mean membrane deformation $R_{m}^{\text {mem }}$ is calculated in analogy to Eq. 1. In addition to the mean membrane deformation $R_{m}^{m e m}$, a cumulative membrane deformation $R_{0.9}^{m e m}$ defining the height in $\mu \mathrm{m}$ at which $90 \%$ of the total membrane is deformed is introduced for determining an interfacial contact area of PTL and CL. At higher values cumulative membrane deformation increases in an asymptotic fashion. XTM reveals that the membrane creeps in pores and is not in contact with PTL particles. Thus $R_{m}^{P T L}$ and $R_{m}^{\text {mem }}$ are interlinked. Low $R_{m}^{P T L}$ indicates smooth PTL surfaces and corresponds to low mechanical membrane deformation. 


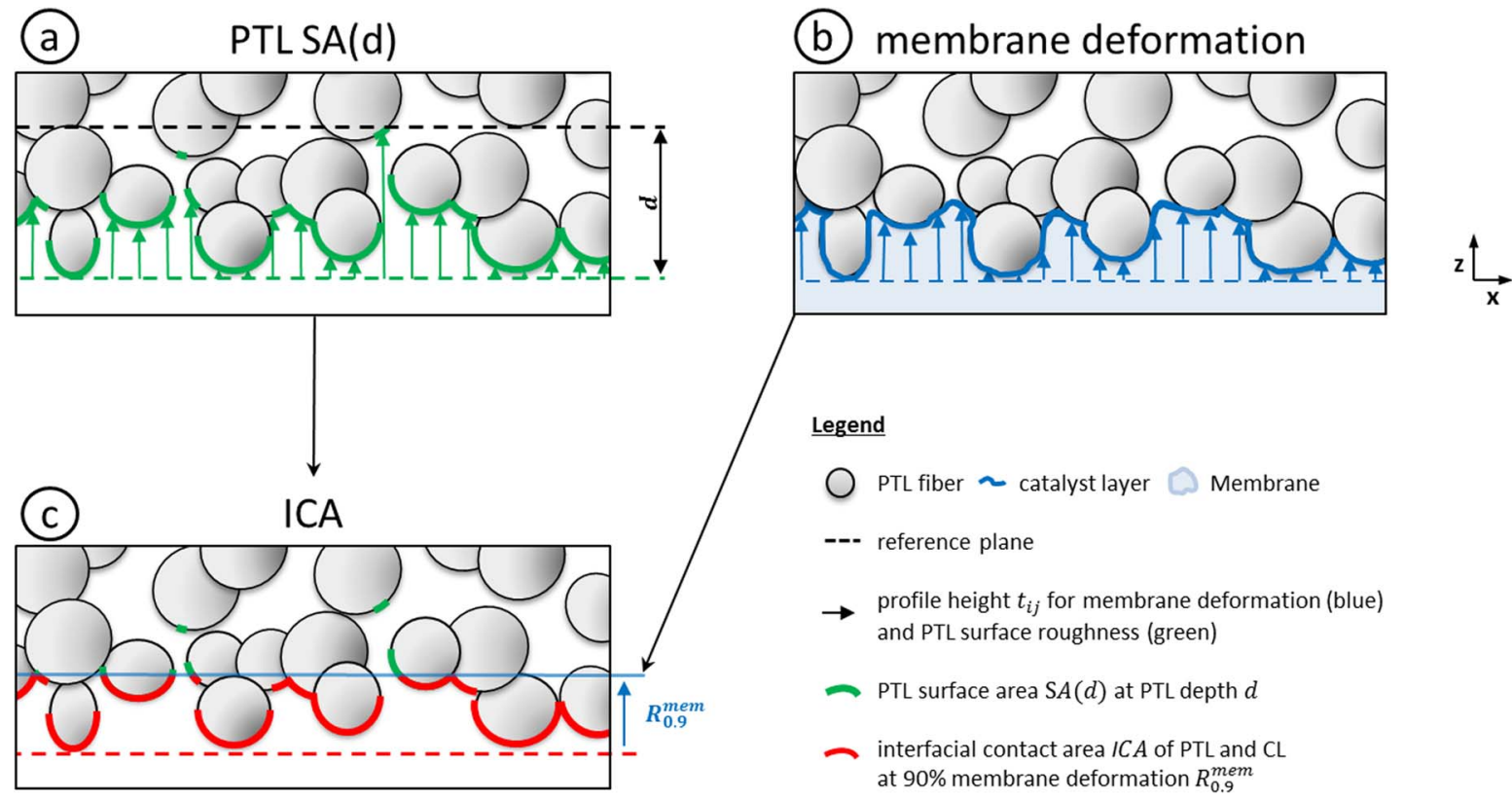

Figure 2. Schematic description of 3D surface analysis: the profile height $t$ is determined from tomography data a) for the PTL resulting in the surface area and b) for the membrane/catalyst layer deformation; arrows indicate local surface/membrane profile height; dashed lines represent the reference planes perpendicular to the height vectors; c) shows the interfacial contact area at a membrane deformation at $90 \%$ depth in red silhouette lines.

Three more conventional amplitude based parameters are introduced to enable comparisons to other PTL materials in future work:

$$
R_{a}=\frac{1}{M N} \sum_{i=0}^{M-1} \sum_{j=0}^{N-1}\left|\Delta t_{i j}\right| \text { with } \Delta t_{i j}=t_{i j}-R_{m}
$$

$R_{a}$ is the arithmetic mean surface roughness. It is based on the mean deviation height of each point from the mean profile height of the total surface $\Delta t_{i j}$, with $\mathrm{M} \times \mathrm{N}$ the number of surface points.

The root mean square roughness $R_{q}$ is the statistical standard deviation of the surface heights:

$$
R_{q}=\sqrt{\frac{1}{M N} \sum_{i=0}^{M-1} \sum_{j=0}^{N-1}\left(\Delta t_{i j}\right)^{2}}
$$

and $R_{\max }$ represents the absolute maximum profile height:

$$
R_{\max }=\max \left(t_{i j}\right)
$$

Specific surface roughness.-In contrast to amplitude roughness, which is based on 1D distances, the hybrid surface roughness is based on the $2 \mathrm{D}$ characteristics of the surface. In analogy to the roughness factor $r f$ used in electrochemical catalysis describing the electrochemical active surface per geometric area, a specific surface area $\mathrm{R}_{S A}(\mathrm{~d})$ is introduced:

$$
\mathrm{R}_{S A}(\mathrm{~d})=\frac{\sum_{i=0}^{M-2} \sum_{j=0}^{N-2} A_{i j}(d)}{(M-1) * \delta_{x} *(N-1) * \delta_{y}}=\frac{S A(d)}{A_{\text {geo }}}
$$

$\mathrm{R}_{S A}(\mathrm{~d})$ is defined as the ratio of the surface area (SA), which is the sum of all surface areas $\mathrm{A}_{\mathrm{ij}}$ at a defined PTL depth $d$ normalized with the geometric area $\mathrm{A}_{\text {geo }}$ where $\delta$ represents the geometric sampling length in $\mathrm{x}$ and $\mathrm{y}$ direction.

Methodology of XTM roughness analysis.-The methodology for the quantification of the interference of PTL and CL is shown in Figure 2. To extract the specific interfacial contact area $\mathrm{R}_{\mathrm{ICA}}$ at the interface of PTL and CL based on post-operando data, surface roughness $\mathrm{R}_{\mathrm{m}}^{\mathrm{PTL}}$ and specific surface area $\mathrm{R}_{\mathrm{SA}}(\mathrm{d})$ of the dry PTL structures are determined according to Eqs. 1 and 5. The SA is highlighted in green silhouette lines where PTL surface heights are given by green arrows in Figure 2a. The mean membrane deformation $\mathrm{R}_{\mathrm{m}}^{\mathrm{mem}}$ and cumulative membrane deformation $\mathrm{R}_{0.9}^{\mathrm{mem}}$ are calculated in the second step.
The two values are determined based on the membrane deformation heights represented by the blue arrow in Figure $2 b$.

From $R_{0.9}^{m e m}$ and $\mathrm{R}_{\mathrm{SA}}(d)$ a specific interfacial contact area $\mathrm{R}_{I C A}$ is then determined as:

$$
R_{I C A}=R_{S A}\left(R_{0.9}^{m e m}\right)
$$

describing the interface area between PTL and CL normalized to the geometric area. Under operating conditions, not the entire available PTL surface is in contact with the catalyst layer which would require extensive membrane deformation of $>60 \mu \mathrm{m}$ depending on the PTL type. Figure $2 \mathrm{c}$ shows the surface utilization marked by a red contour line while the residual, non-contacted surfaces is shown in green.

Computing of the roughness parameters.-The surface properties of the PTL materials were analyzed by an in-house developed 3D XTM roughness analysis method. The reference coordinate system is defined as shown in Figure 3, where $x$ represents the PTL width, y and $\mathrm{z}$ the length and thickness. The XTM analysis is performed on through-plane CT stacks comprising of a defined number of CT slices $i$. The first $\mathrm{x}-\mathrm{y}$ slice containing a solid voxel is defined as the reference plane. The length of the vector normal to the reference plane represents the profile height $t_{i j}$ at position $j$ in $\mathrm{x}$ direction. The roughness analysis is conducted for $\mathrm{N}$ positions and $\mathrm{M}$ stack slices. The sampling length $\delta$ is given by the XTM voxel cube edge length. To determine the PTL surface area, a polyhedral surface meshing is then applied based on the surface matrix in Matlab.

\section{Results}

The goal of this work is to correlate the PTL bulk and surface properties with the performance in PEWE. A systematic approach enables the elucidation of poorly understood transport processes in PEWE.

A matrix of 6 PTL materials consisting of 3 fiber diameters (labeled as $1,2,3$ ) and two nominal porosities of $56 \%$ (labeled as L) and $76 \%$ (labeled as $\mathrm{H}$ ) was characterized. L1 refers therefore to a fiber based PTL having a nominal fiber diameter of $14 \mu \mathrm{m}$ and a porosity of $56 \%$. All materials have a thickness of $1 \mathrm{~mm}$. 3D-renderings of cubes with an edge length of $0.6 \mathrm{~mm}$ are given in Figure 1, with the Ti solid phase shown in gray. A commercially available state of the art powder 

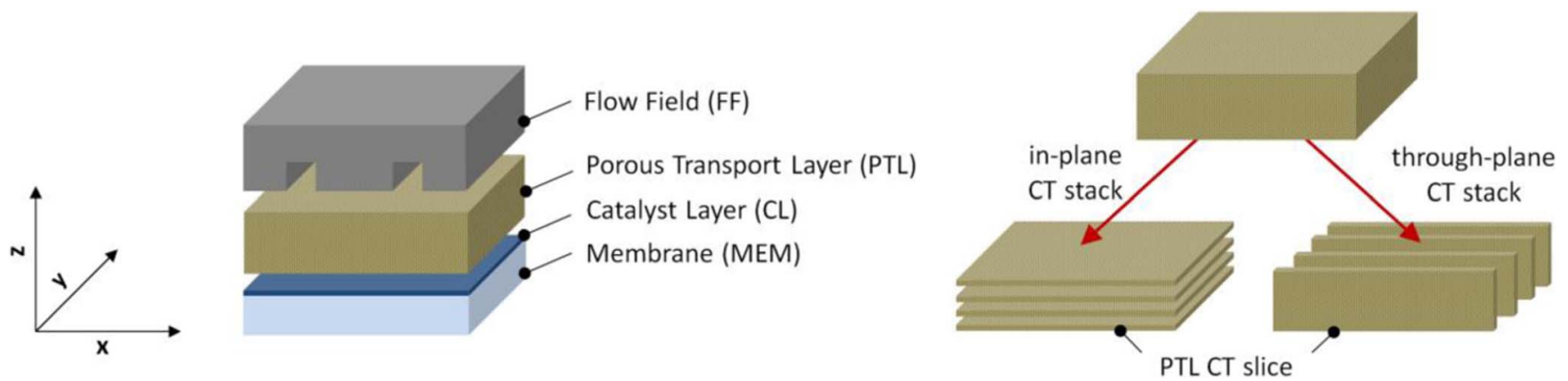

Figure 3. The simplistic cartoon shows the reference coordinate system based on the cross-section of a half cell as well as the computed tomography (CT) stacks of a PTL; the $x-y$ plane are considered as the in-plane direction, $z$-x plane as through-plane direction.

sintered material, SIKA T10 (Sinter Metals Filters GKN, Germany) was used as reference material for comparison.

The two main materials properties, morphology (bulk properties) and topology (surface properties) are discussed in the following sections. The first part focusses on the bulk properties and the related transport parameters with REVs (based on porosity) determined for all materials. In the second part the surface properties of the materials were analyzed. The surface roughness of PTLs as well as the membrane deformation were computed for the different materials. Subsequently the characterization of the interfacial surface between PTL and catalyst layer was performed.

\section{Morphology Characterization}

Representative elementary volume (REV).-To ensure that the structural investigations are based on a reliable volume domain, the representative elementary volume was determined for the porosity. The maximum number of cubes with given cube edge lengths of $100 / 200 / 400 / 600 / 900 \mu \mathrm{m}$ were fitted in the bulk ROI of $2000 \times 1000$ $\times 900 \mu \mathrm{m}^{3}$. The statistics related to the porosity of the population of the inserted cubes was then evaluated. A standard deviation of $<3 \%$ for the porosity of the cube population was defined as criteria for reaching REV. Detailed data are given in Supplementary Table I. The results exemplify the dependency of the REV on porosity and feature size. The REV size grows when increasing fiber diameters from $200 \mu \mathrm{m}^{3}$ (L1) to $600 \mu \mathrm{m}^{3}$ (L3). An increase of porosity to $76 \%$ also results in higher REVs of $400 \mu \mathrm{m}^{3}$ (H1) to $900 \mu \mathrm{m}^{3}$ (H3) (see Table I). The ROIs then used to determine porosities and pore/particle size distributions $(900$ $\left.\times 1000 \times 2000 \mu^{3}\right)$ and transport parameters $\left(600 \mu \mathrm{m}^{3}\right.$ and 900 $\mu \mathrm{m}^{3}$ ) were chosen significantly larger than the REVs determined for porosity to avoid ambiguity. The ROIs used for the quantification are reported in footnotes of the Tables for transparency.

Porosity.-The XTM determined porosity values $\varepsilon_{\mathrm{XTM}}$ of $55 \pm 1 \%$ (L1), $54 \pm 1 \%$ (L2), and $57 \pm 1 \%$ (L3) for the low and $75 \pm 1 \%(\mathrm{H} 1)$, $74 \pm 1 \%(\mathrm{H} 2)$, and $78 \pm 2 \%(\mathrm{H} 3)$ for the high porosity materials are well in line with the nominal porosities of $56 \%$ and $76 \%$, respectively. The XTM based porosities also agree well with porosities obtained from gravimetric measurements $\varepsilon_{\text {grav }}$. The maximum standard deviation between both porosities is determined to be $2 \%$ for the matrix.

Continuous pore and particle size distribution (cPSD).-Continuous pore and particle size distributions (cPSD) were determined for all six samples. cPSD provides the statistical characteristics for bulk properties of the materials. As these distributions are determined independent of direction and location they are labeled continuous PSD. ${ }^{29}$ The resulting histograms for both, solid size distributions (SSD) and void size distribution (VSD) are shown in Figure 4. All histograms are fitted based on a Lorentzian approach for statistical comparison.

Table I. Morphological properties of the Ti fiber and Ti sinter PTL materials as porosity $\varepsilon$, pore $d_{\text {pore }}$ and particle diameter $d_{f i b r e}$ as well as pore diameters computed by mercury intrusion porosimetry $d_{M I P, 50}$ and continuous PSD $d_{c P S D, 50}$ at $50 \%$ cumulative pore volume were analyzed based on ROI $\geq$ REV. The parameters tortuosity $\tau$, structural factor $f$, effective permeability $\kappa$, effective thermal $\sigma_{t h e r m}$ and electrical conductivities $\sigma_{\text {elec }}$ were computed for the orthogonal space $\mathrm{x} / \mathrm{y} / \mathrm{z}$ directions, where $\mathrm{z}$ is the through plane direction and $\mathrm{x}$ and $\mathrm{y}$ the Cartesian in-plane directions. T10 parameters marked by ${ }^{*}$ were extracted from Suermann et al. ${ }^{5}$ for comparison.

\begin{tabular}{|c|c|c|c|c|c|c|c|c|}
\hline PTL type & unit & L1 & $L 2$ & $L 3$ & $H 1$ & $H 2$ & $H 3$ & $T 10$ \\
\hline$R E V$ & \multicolumn{8}{|c|}{ based on XTM dry PTL structure } \\
\hline REV length & $\mu m$ & 200 & 400 & 600 & 400 & 400 & 900 & $400^{*}$ \\
\hline$\varepsilon_{X T M}$ & $\%$ & $55 \pm 1$ & $54 \pm 1.0$ & $57 \pm 1$ & $75 \pm 1$ & $74 \pm 1$ & $78 \pm 2$ & $35 \pm 2^{*}$ \\
\hline Morphology ${ }^{1}$ & \multicolumn{8}{|c|}{ based on XTM dry PTL structure } \\
\hline$d_{\text {pore }}$ & $\mu m$ & $17.8 \pm 0.2$ & $20.1 \pm 0.3$ & $51.3 \pm 0.8$ & $32.4 \pm 0.6$ & $45.5 \pm 0.6$ & $123.6 \pm 1.0$ & $38^{*}$ \\
\hline$d_{\text {fiber }}$ & $\mu m$ & $11.0 \pm 0.1$ & $15.4 \pm 0.2$ & $30.1 \pm 0.2$ & $11.1 \pm 0.1$ & $17.4 \pm 0.2$ & $29.3 \pm 0.1$ & $52^{*}$ \\
\hline$d_{c P S D, 50}$ & $\mu m$ & $20.6 \pm 0.2$ & $23.5 \pm 0.3$ & $58.0 \pm 0.4$ & $36.4 \pm 0.3$ & $45.8 \pm 0.3$ & $103.1 \pm 0.3$ & $35.5 \pm 0.3$ \\
\hline$d_{M I P, 50}$ & $\mu m$ & $13.9 \pm 0.0$ & $16.9 \pm 0.1$ & $42.5 \pm 0.3$ & $27.6 \pm 0.2$ & $36.5 \pm 0.3$ & $78.2 \pm 0.8$ & $25.9 \pm 0.2$ \\
\hline Transport $x / y / z^{2}$ & \multicolumn{8}{|c|}{ based on XTM dry PTL structure } \\
\hline$\tau$ & - & 1.7/1.6/1.6 & $1.6 / 1.6 / 1.5$ & $1.3 / 1.3 / 1.3$ & 1.3/1.2/1.2 & 1.2/1.2/1.2 & $1.1 / 1.1 / 1.2$ & $/ / 1.6^{*}$ \\
\hline$f=\frac{\varepsilon}{\tau^{2}} 10^{2}$ & - & $19 / 23 / 23$ & $24 / 22 / 27$ & $34 / 33 / 33$ & $48 / 51 / 52$ & $50 / 48 / 53$ & $60 / 64 / 57$ & $/ / 14^{*}$ \\
\hline$\kappa 10^{-12}$ & $m^{2}$ & $1.7 / 2.2 / 2.4$ & $3.7 / 3.3 / 5.0$ & $26.9 / 24.3 / 30.2$ & $17.4 / 19.4 / 21.0$ & $31.9 / 29.2 / 39.4$ & $150.5 / 186.3 / 156.8$ & $/ / 3.4^{*}$ \\
\hline$\sigma_{\text {elec }} 10^{5}$ & $S / m$ & $5.6 / 6.4 / 5.4$ & $5.9 / 5.5 / 5.2$ & $5.8 / 5.7 / 3.6$ & 2.6/2.9/1.1 & $3.1 / 2.9 / 1.4$ & $2.2 / 3.0 / 0.6$ & $/ / 9^{*}$ \\
\hline$\sigma_{\text {therm }}$ & $W / m K$ & $6.0 / 6.6 / 5.9$ & $6.2 / 5.9 / 5.8$ & $6.1 / 6.1 / 4.5$ & $3.1 / 3.5 / 2.1$ & $3.6 / 3.5 / 2.4$ & $2.9 / 3.5 / 1.7$ & $/ / 9.6^{*}$ \\
\hline
\end{tabular}

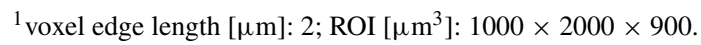

${ }^{2}$ voxel edge length $[\mu \mathrm{m}]: 2$; ROI $\left[\mu \mathrm{m}^{3}\right]: 600$ (L1, L2, H1, H2), 900 (L3, H3). 

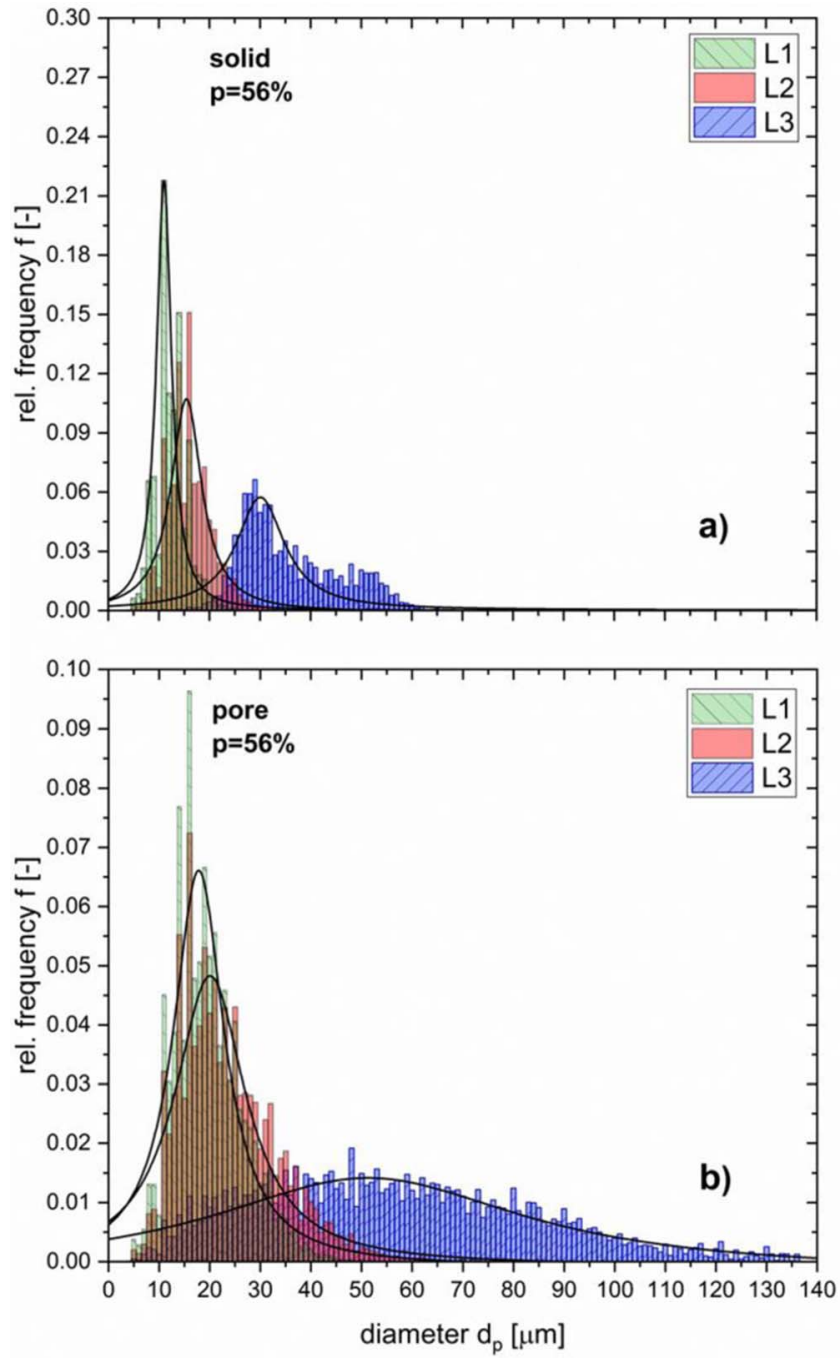
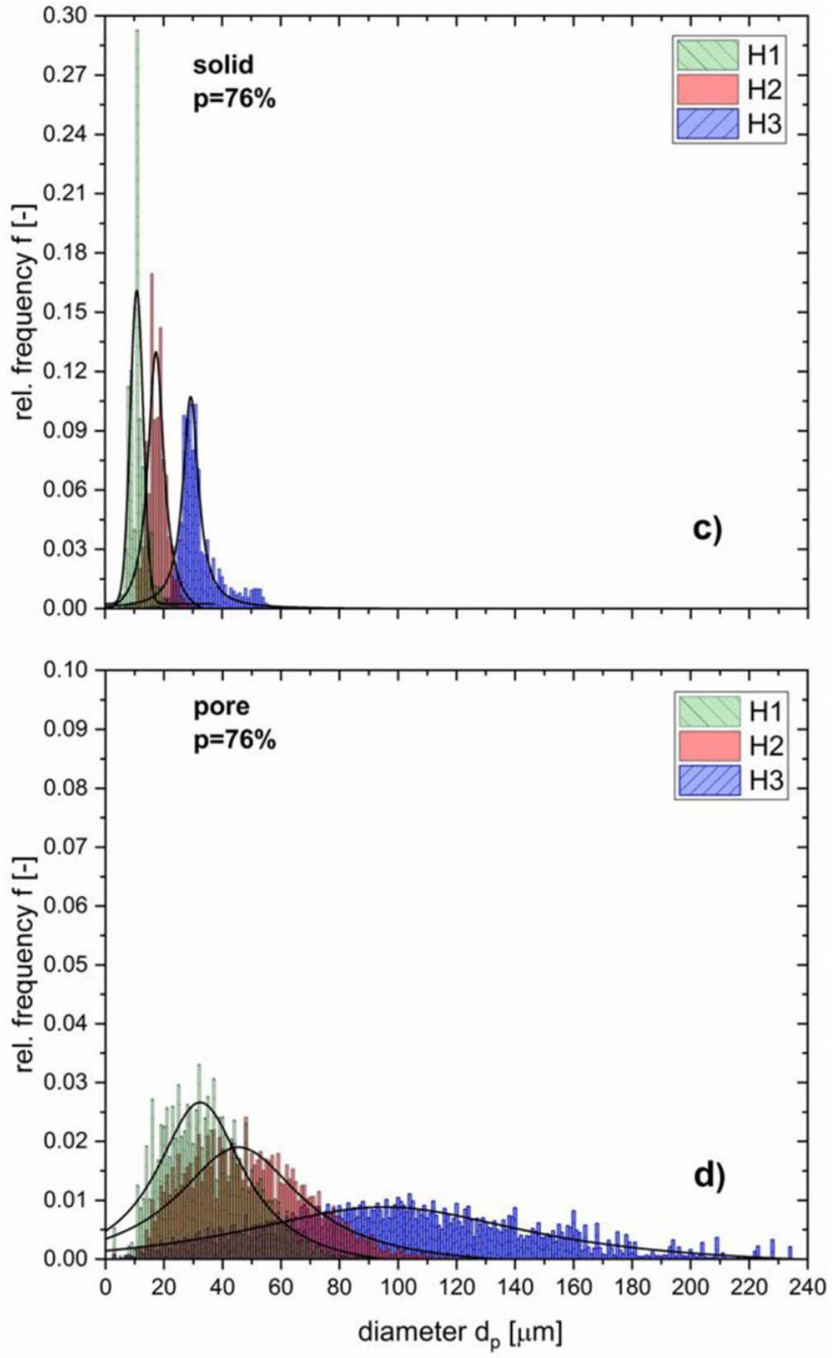

Figure 4. a) and c) show the particle size distribution for fiber types 1 to 3 at a constant porosities of 56\% and $76 \%$; b) and d) represent the void size distribution.

The L1/L2 and H1/H2 materials based on the thinner fibers show narrow SSDs. The fiber diameters have a narrow spectrum in the range of 5-35 $\mu \mathrm{m}$ with mean diameters of $11 \mu \mathrm{m}$ and 15-17 $\mu \mathrm{m}$, respectively (see Table I). Fiber 3 type materials L3 and H3 feature a broader distribution with a mean fiber diameter of $30 \mu \mathrm{m}$. The narrow SSDs provide a controlled insight in governing structure parameters, contrary to state of the art particle sinter materials such as T10, featuring broad PSDs from $2 \mu \mathrm{m}$ up to $200 \mu \mathrm{m}^{5}$.

The VSDs show identical trends to the SSDs, with sharp peaks for pore spectrums of the materials of fiber types 1 and 2 . For L1 and L2 pore diameters are in the range of $5 \mu \mathrm{m}$ to $60 \mu \mathrm{m}$, with mean diameters of $18 \mu \mathrm{m}$ and $20 \mu \mathrm{m}$ respectively. L3 has significantly larger pore sizes, i.e. in the range of 5 to $130 \mu \mathrm{m}$ with a mean diameter of $51 \mu \mathrm{m}$. An increase in porosity from $56 \%$ to $76 \%$ results for all three fiber diameters in an approximate doubling of the pore size spectrum with mean diameters shifted to $32 \mu \mathrm{m}, 46 \mu \mathrm{m}$ and $123 \mu \mathrm{m}$ for $\mathrm{H} 1$, $\mathrm{H} 2$ and $\mathrm{H} 3$, respectively.

Mercury intrusion porosimetry PSD (MIP-PSD)._cPSD does not give any information on throats or bottle-necks. However, these bottle-necks may be responsible for mass transport restrictions of the fluids, an effect described well for gas diffusion materials of polymer electrolyte fuel cells for water imbibition ${ }^{37,38}$ and its effects on gas transport. In the case of electrolysis, no direct experimental proof has been shown so far, but several studies propose PTL bulk structure related transport limitations. ${ }^{12,15,28}$ Therefore directed mercury intrusion porosimetry PSD (MIP-PSD) was computed and results are discussed for the PTL thickness direction $\mathrm{z}$.

Figure 5 shows the cPSD and MIP-PSD based cumulative pore volumes for the $\mathrm{L} 2$ and $\mathrm{H} 2$ materials, including sigmoidal curve fits. The results for L2/H2 exemplify the trend for all 6 materials. For pores with small diameters $<8 \mu \mathrm{m}, \mathrm{cPSD}$ and MIP-PSD are almost identical (accessible pore volume $>99 \mathrm{vol} \%$ ) a trend observed for all fiber materials. Detailed data for all materials, fibers and T10 are shown in Supplementary Figure 1.

At larger diameters, different decreases in the cumulative pore volume are observed, as seen in Figure 5. For L2 a doubling of the sphere diameter from 8 to $16 \mu \mathrm{m}$ results in a decrease of the MIP-pore volume to $63 \mathrm{vol} \%$ while for $\mathrm{H} 2$ the accessible volume drops only marginally to $97 \mathrm{vol} \%$. For smaller fibers and low porosity (L1) this transition is more pronounced changing to $12 \mathrm{vol} \%$ at $16 \mu \mathrm{m}$, while an increase in fiber diameter $(\mathrm{L} 3, \mathrm{H} 3)$ results in a slower decrease due to bigger pore sizes. The accessible MIP-pore volumes of L3 and $\mathrm{H} 3$ remain close to $100 \%$ when doubling the sphere diameter from $8 \mu \mathrm{m}$ to $16 \mu \mathrm{m}$.

The difference in the cumulative volumes between cPSD and MIPPSD at the same pore diameter is a consequence of the bottleneck effect imposed by local small connections in the pore matrix. This bottleneck effect becomes dominating if the MIP-PSD cumulative volume differs significantly from the cPSD value. The maximum change of MIP-PSD is given by the inflection point. Bigger pore diameter above mentioned inflection point cause significant differences between MIP-PSD and PSD. A Difference of $>20 \mathrm{vol} \%$ between MIP-PSD and cPSD pore 


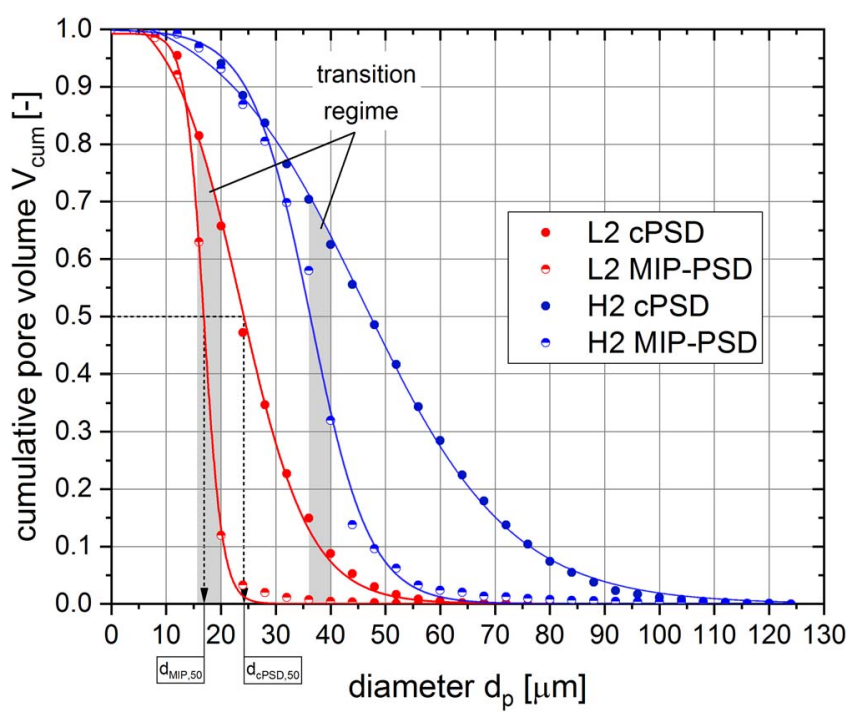

Figure 5. Cumulative pore volumes for cPSD and MIP-PSDs are shown for the materials L2 and H2. Curves are fitted with a sigmoidal function.

volumes at a given diameter captures the transition of available pore space and is therefore defined as the evaluation criteria. In Figure 5, the gray domains indicate the pore sizes where the $20 \%$ criteria is exceeded. The pore sizes were found to be at $14 \mu \mathrm{m}, 18 \mu \mathrm{m}$ and $42 \mu \mathrm{m}$, for the L1, L2 and L3 materials, while the H-materials values are about a factor of 2 higher.

The values of $d_{C P S D, 50}$ and $d_{M I P, 50}$ represent the pore diameters at 50 vol $\%$. The $d_{M I P, 50}$ values for all materials L1 to H3 are close to the values where the bottleneck effect is expected to start. The same trend was also observed for the powder sintered material T10, see Supplementary Information. If bottleneck sizes indeed play a governing role for mass transport in the porous structure and hence for PEWE performance, $d_{M I P, 50}$ could therefore be used as a criteria for PTL design. The increase of porosity from $56 \%(\mathrm{~L})$ to $76 \%(\mathrm{H})$ results in approx- imately a doubling of the $d_{C P S D, 50}$ and $d_{M I P, 50}$ values. The values are listed in Table I.

Fiber orientation.-All six fiber materials show a strong inplane/through-plane anisotropy. It is obvious that the materials have a quasi-2D structure with horizontally stacked fibers as illustrated in Figure 1. However also for the in-plane fiber orientation, anisotropy affects transport properties. Therefore a $2 \mathrm{D}$ analysis for fiber orientation, plotted in polar coordinates, is shown in Figure 6 with sub-plots for the $\mathrm{L}$ and $\mathrm{H}$ materials. Figure 6 exemplifies that all materials feature preferential fiber orientations rather than random distributions. The fiber type materials L1, L2 and L3 show fibers arranged in $\sim 45^{\circ}$ intervals. The increase in porosity ( $\mathrm{H}$ samples) results in less preferential fiber distributions. Especially $\mathrm{H} 3$ shows narrow peaks in the range of $20^{\circ}$ to $-30^{\circ}$. The small difference in fiber orientation between the fibers itself $\left(<\Delta 90^{\circ}\right)$ appears to have an impact on in-plane conductivity. The fiber orientation properties are compared to the in-plane transport properties in the next section.

Transport parameters.-Different PTL transport properties in the void and solid phases are important for PEWE operation..$^{24,39,40}$ When a channel based flow field is used, then the through-plane properties will dominate species transport in the channel areas while a mixture of through-plane and in-plane properties is relevant for the rib areas. For energy transport the situation is inversed as heat will flow through the flow field ribs. Therefore, analysis of through-plane properties is discussed first followed by in-plane properties.

The transport of species in porous media is dominated by concentration gradients for effective diffusivity as well as by pressure for permeability. The bulk diffusion in porous media $\mathrm{D}_{\text {eff }}$ can be described according to Equation 7 where $\mathrm{D}^{0}$ is the diffusion coefficient in empty space and $f$ a structural factor defined as the quotient of the porosity $\varepsilon$ and the square of the tortuosity $\tau:^{41}$

$$
D_{e f f}=D^{0} * f \text { with } f=\frac{\varepsilon}{\tau^{2}}
$$

Through-plane tortuosities for the fiber based PTLs are in the range of 1.2 to 1.6 with the general trend that smaller fiber diameters and lower porosities result in higher tortuosity values, see Table I. The
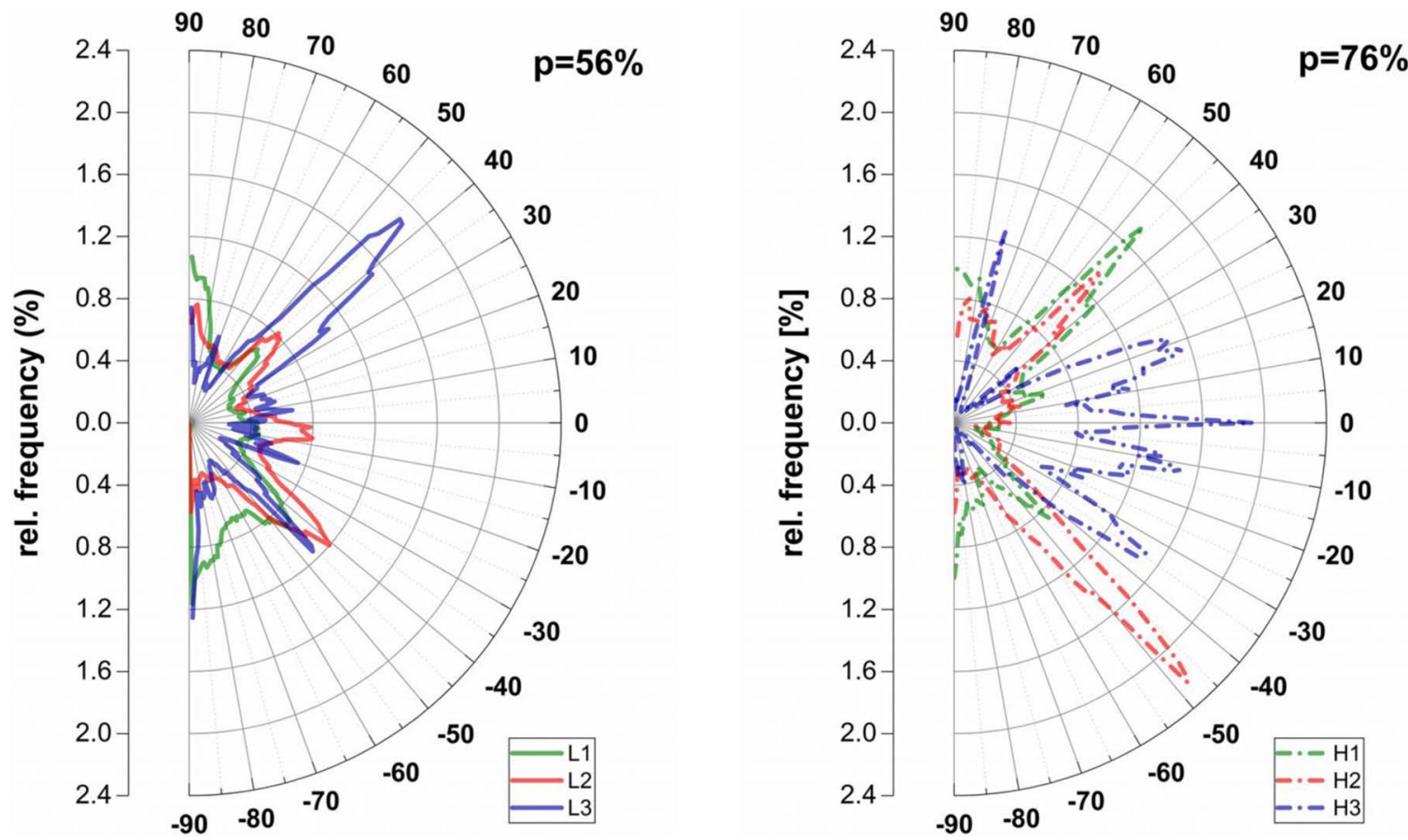

Figure 6. The relative frequency of the in-plane fiber orientation is plotted in a polar coordinate diagram from $90^{\circ}$ to $-89^{\circ}$ for the three fiber types for $56 \%$ and $76 \%$ porosity. 

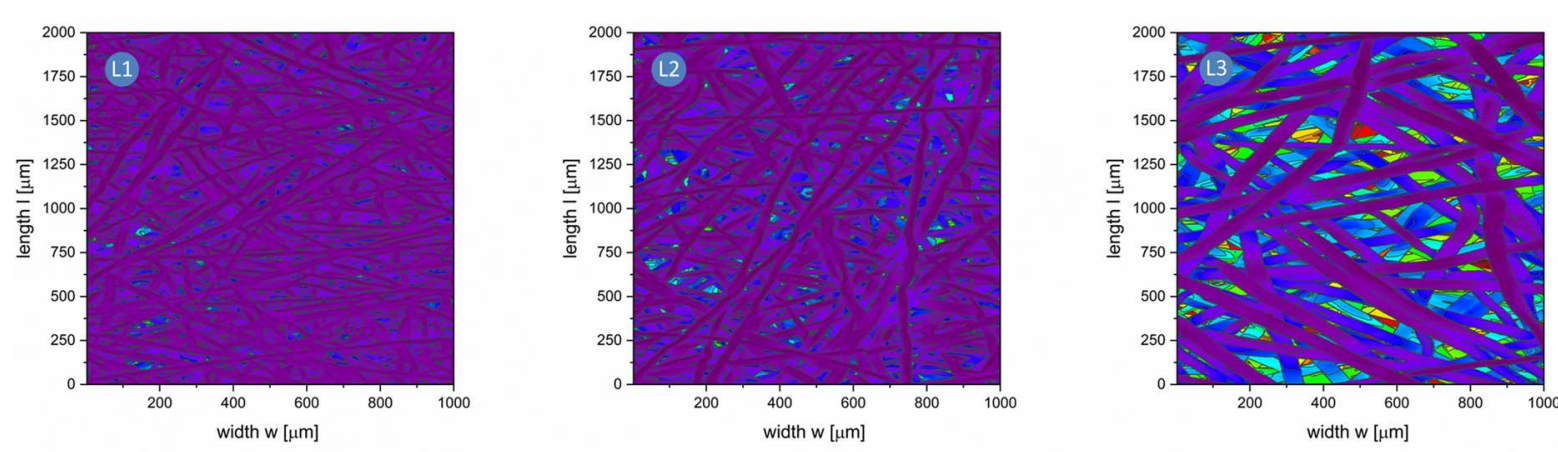

profile height $t[\mu \mathrm{m}]$
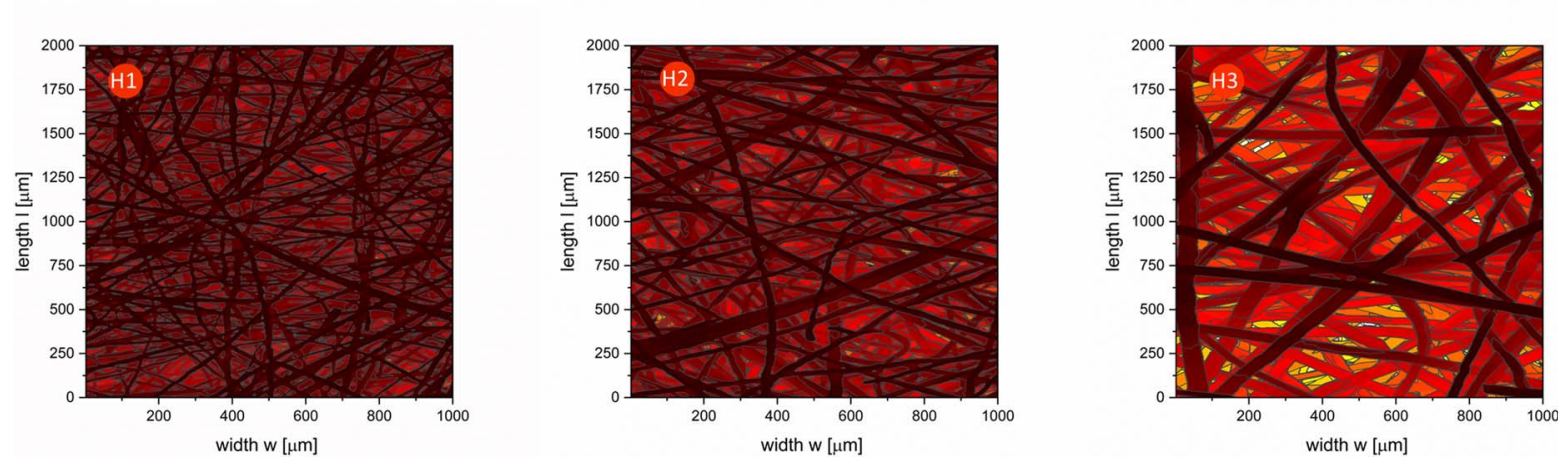

profile height $t[\mu \mathrm{m}]$

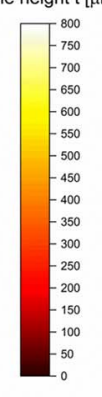

Figure 7. The roughness profile height maps for all six fiber based PTLs are shown. The profile heights are represented by color scales.

particle sintered material has a tortuosity of 1.6 which equals the value of sample L1 having the smallest fiber size and low porosity. The through-plane effective diffusivity trends are therefore the same, but more pronounced due to the power dependence on $\tau$. The throughplane structural factor $f$ almost triples from 0.23 to 0.57 with increasing mean fiber size and porosity, see Table I. As compared to T10, all fiber materials feature higher effective through-plane diffusivities (a factor of 1.6 to 4 ) due to their higher porosity and lower tortuosity.

The permeability of porous materials proposed as PTL structures for PEWE in the literature shows differences up to 2 orders in magnitude with values from $10^{-12} \mathrm{~m}^{2}$ to $10^{-10} \mathrm{~m}^{2}{ }^{24}$ The fiber materials also have a wide permeability spectrum, ranging from $157 \times 10^{-12} \mathrm{~m}^{2}$ for $\mathrm{H} 3$ to $\mathrm{L} 1$ with $3 \times 10^{-12} \mathrm{~m}^{2}$, being the only fiber material featuring a similarly low permeability as T10.

The transport properties in the solid phase are important for energy transport, i.e. cooling. Low porosity and small fiber diameters provide better heat transport properties and lower electric resistivity. This is due to higher numbers of contacts between the horizontally oriented fibers. Materials with the thin fibers of type 1 and 2 show similar conductivities in the solid due to small differences in fiber diameters. The through-plane thermal conductivity of all fiber materials (1.7 to 5.9 $\mathrm{W} /(\mathrm{mK}))$ is lower than the one for the T10 powder sinter material with $9.6 \mathrm{~W} /(\mathrm{mK})^{5}$ due to the comparably low porosity of the T10 material. There is a subtle trade-off between the transport properties in the solid (heat) and the void (fluids). This is a significant difference to polymer electrolyte fuel cells, where, due to lower heat flux densities, upper limits for the porosity seem not to be limited by the heat transport.

Despite different in-plane orientation of the fibers, there was no significantly different permeability observed between the in-plane and through-plane directions for all fiber materials. The relatively large porosities, big pore sizes as well as quasi-uniform tortuosity in the different directions appear to cause uniformity. Generally a factor of two for permeability is obtained for increasing the porosities from $56 \%$ to $76 \%$ for the same fiber types.

In contrast, the solid transport properties of thermal and electrical conductivities are influenced by the non-homogenous distribution of the fiber directions (see Figure 6). PTLs with broader polar fiber distributions show minor differences $<10 \%$ in in-plane vs. through-plane conductivities. $\mathrm{H} 3$ featuring the least uniform radial fiber distribution has significantly higher differences between in-plane and trough-plane electrical $(17 \%)$ and thermal $(27 \%)$ conductivities

\section{Topology Characterization}

Not only the bulk properties, but also the surface properties of the PTL materials affect PEWE performance and durability. It is therefore of interest to quantify PTL surface properties in order to characterize their mechanical and electrochemical interaction with the catalyst layer and the membrane. A high surface roughness can cause irreversible structural changes to the membrane electrode assembly in form of plastic deformation as reported previously. ${ }^{7}$ Applied mechanical stress triggers degradation in long term PEWE operation as demonstrated by Stucki et al. ${ }^{1}$ Crack formation in the catalyst layer as well as local mechanical membrane thinning causing increased gas cross-over is observed. An unsuitable surface may therefore prevent the use of thin membranes for high pressure and high power density PEWE applications.

Furthermore, a fundamental understanding of the impact of the $\mathrm{PTL} /$ catalyst layer interface on electrochemical performance is required. Lower ohmic resistances have been observed for T10 modified with vacuum plasma sprayed layers, ${ }^{11}$ but the topic is only scarcely addressed in literature. It is therefore necessary to correlate the mass transport and kinetics overpotentials to the PTL/CL interface. The PTL topology is identified to be the governing parameter affecting all overpotentials. The correlation is discussed in the second paper of the series.

PTL surface roughness.-Profile height maps of L1 to H3 are shown in Figure 7. In all maps, fibers with length $>2 \mathrm{~mm}$ can be observed, crossing the entire ROI. The 2D structure of in-plane stacked fiber layers can be clearly identified. For comparison, the T10 profile height map is given in Supplementary Figure 2.

The fiber sizes have a significant impact on the fiber distance distribution. Smaller fibers materials of type 1 and 2, feature finely distributed fibers with small pores and short distances between the single fibers $(20 \mu \mathrm{m}-30 \mu \mathrm{m})$. L3 and H3 materials show significantly increased separation. Also when increasing the porosity from $56 \%$ to $76 \%$, within the first $50 \mu \mathrm{m}$ of depth the number of fibers and therefore 

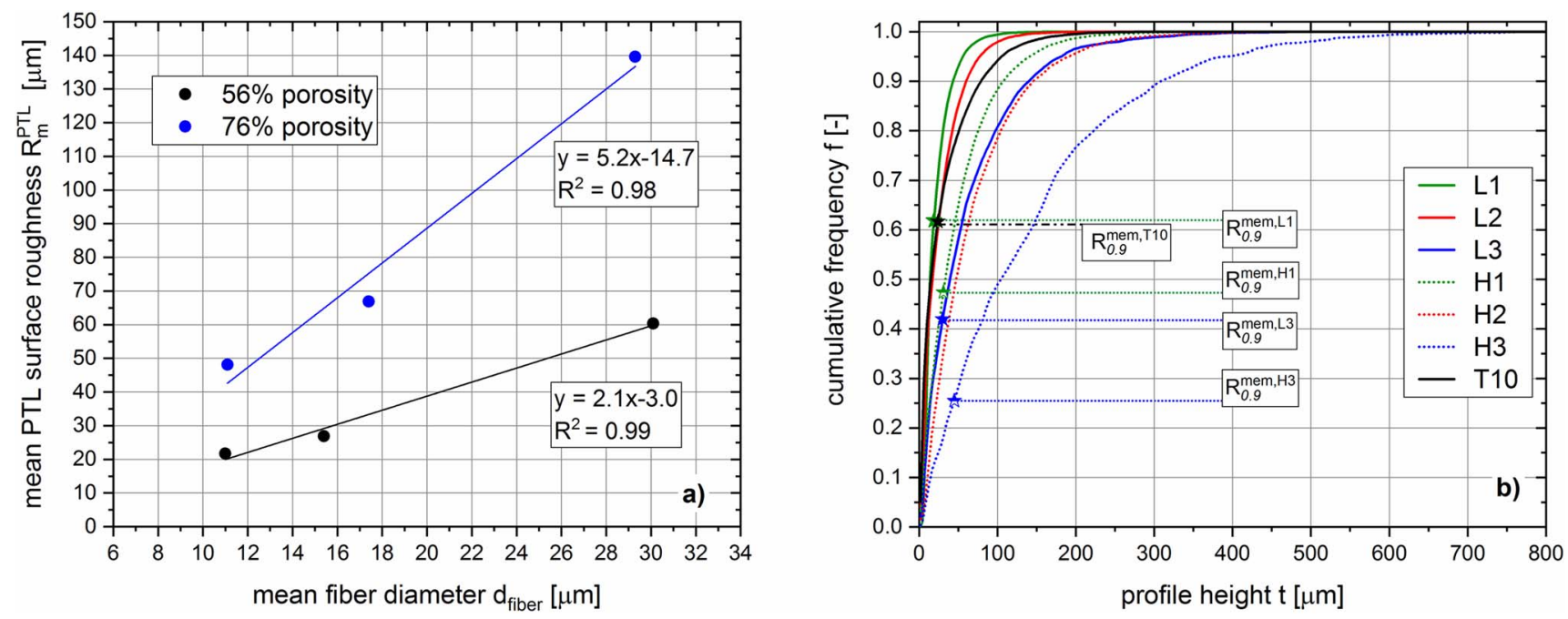

Figure 8. Sub-plot a) shows mean surface roughness $R_{m}^{P T L}$ as function of mean fiber diameter. Cumulative frequency of surface height is plotted for every PTL material in sub-plot b. Membrane deformations $R_{0.9}^{m e m, i}$ obtained from cell measurements with tested PTL $i$ are included for comparison and represented by asterisks.

related contact area to the CL reduces drastically, see next section. The distances between the contact areas of PTL and CL, given by the fiber distances may play a significant role for catalyst layer utilization and ohmic interfacial resistances because of the relatively high electric and ionic catalyst layer resistance. ${ }^{25,42}$ Contrary wise, with the increase of porosity and solid feature sizes may promote fluid transport if permeability is important. A consequence of high porosity and large fibers is an increase of maximal surface height $R_{\max }$. Values for $R_{\max }$ of 498 $\mu \mathrm{m}$ and $798 \mu \mathrm{m}$ are observed for L3 and H3, respectively with direct fluid flow paths penetrating up to $50 \%$ and $80 \%$ of the PTL thickness without diversion.

Fiber thickness is also correlated to the surface roughness $R_{m}^{P T L}$. Figure 8a shows an approximately linear dependence at constant porosities. This empirical relation of the linear iso-porosity lines enables a simple determination of PTL surface properties based on preknowledge of the mean fiber diameter. The mean surface roughness
$R_{m}^{P T L}$ correlates with porosity. $R_{m}^{P T L}$ increases by $57 \% \pm 2 \%$ when the porosity is raised from $56 \%$ to $76 \%$ independent of the fiber type, all values are listed in Table II.

Figure $8 \mathrm{~b}$ shows the cumulative frequency of surface height $t$. The plot can be interpreted as follow: $50 \%$ of the PTL surface of $\mathrm{H} 3$ can be contacted by a tensile instrument screening the PTL surface with a tip with a height of $100 \mu \mathrm{m}$ whereas $90 \%$ of the surface is accessible for a tip with a height of $300 \mu \mathrm{m}$. Approximately linear behavior is found up to $90 \%$ followed by an asymptotic plateau. As described above, increasing fiber diameter and related pore size growth enhances the roughness of a material. T10, despite coarse solid particles, shows a relatively low surface roughness due to its low porosity of $35 \%$.

In Figure $8 \mathrm{~b}$ also the contact depth of the membrane, determined by the membrane deformation $R_{0.9}^{\text {mem }}$ (see section below) are indicated by asterisks. The membrane deformations lie in the range of $40 \%$ to $60 \%$ of the PTL surface height. H3 is an exception. Only a slight low

Table II. Topological properties of the six Ti-fiber based PTL materials L1 to H3 and Ti-powder sintered material T10 as well as the membrane deformation values are listed in the table. The mean surface roughness $R_{m}$, surface roughness at $90 \%$ cum $R_{0.9}$, maximum surface roughness $R_{\text {max }}$, arithmetic mean surface $R_{a}$, root mean square roughness $R_{q}$ of PTLs, respectively membrane deformation, were calculated from XTM analysis. The interface sub-table contains the equivalent depth $d_{e q}$ at a specific surface area of $1 \mathrm{~m}^{2} / \mathrm{m}^{2}$ geo, the specific surface area $R_{S A}$ at 10 um depth as well as the specific interfacial contact area $R_{I C A}$ defining the actual contact area between PTL and CL under PEWE operation.

\begin{tabular}{|c|c|c|c|c|c|c|c|c|}
\hline PTL type & unit & $L 1$ & $L 2$ & $L 3$ & $H 1$ & $H 2$ & $H 3$ & $T 10$ \\
\hline Mem deformation $^{1}$ & & \multicolumn{7}{|c|}{ based on post-operando catalyst layer/membrane deformation } \\
\hline$R_{m} \pm s t d$ & $\mu m$ & $11.7 \pm 6.3$ & & $16.6 \pm 8.9$ & $18.8 \pm 8.7$ & & $26.2 \pm 14.3$ & $18.3 \pm 6.2$ \\
\hline$R_{0.9}$ & $\mu m$ & 19 & & 30 & 31 & & 45 & 24 \\
\hline$R_{\max }$ & $\mu m$ & 30.0 & & 47.6 & 44.8 & & 57.0 & 33.0 \\
\hline \multicolumn{4}{|l|}{ PTL roughness ${ }^{2}$} & \multicolumn{4}{|c|}{ based on XTM dry PTL structure } & \\
\hline$R_{m} \pm s t d$ & $\mu m$ & $21.7 \pm 17.6$ & $26.9 \pm 25.9$ & $60.4 \pm 62.9$ & $48.1 \pm 46.7$ & $66.9 \pm 61.8$ & $139.6 \pm 125.2$ & $30.9 \pm 36.7$ \\
\hline$R_{0.9}$ & $\mu m$ & 40.0 & 60.0 & 140.0 & 108.0 & 146.0 & 308.0 & 80.0 \\
\hline$R_{a} \pm R_{q}$ & $\mu m$ & $12.7 \pm 12.2$ & $19.4 \pm 17.2$ & $45.7 \pm 43.2$ & $34.3 \pm 31.6$ & $45.6 \pm 20.9$ & $96.0 \pm 80.4$ & $26.6 \pm 25.6$ \\
\hline$R_{\max }$ & $\mu m$ & 256.0 & 278.0 & 498.0 & 636.0 & 498.0 & 798.0 & 452.0 \\
\hline \multicolumn{9}{|c|}{ ased on XTM dry PTL structure and post-operando catalyst layer/membrane deformation } \\
\hline$d_{e q} @ R_{S A}=1$ & $\mu m$ & 8.9 & 10.8 & 26.3 & 18.9 & 31.6 & 58.3 & 10.4 \\
\hline$R_{S A}(10 \mu \mathrm{m})$ & $m^{2} / m_{g e o}{ }^{2}$ & 1.14 & 0.95 & 0.42 & 0.49 & 0.27 & 0.18 & 0.98 \\
\hline$R_{I C A}$ & $\mathrm{~m}^{2} / \mathrm{m}_{\text {geo }} 2$ & 1.81 & $1.60^{*}$ & 1.11 & 1.57 & $1.00^{*}$ & 0.76 & 1.67 \\
\hline \multicolumn{9}{|c|}{$\begin{array}{l}{ }^{1} \text { voxel edge length }[\mu \mathrm{m}]: 3(\mathrm{~L} 1, \mathrm{H} 3), 2.8(\mathrm{~L} 3, \mathrm{H} 1) ; \mathrm{ROI}[\mu \mathrm{r} \\
(\mathrm{T} 10) \text {. } \\
{ }^{2} \text { voxel edge length }[\mu \mathrm{m}]: 2 ; \mathrm{ROI}\left[\mu \mathrm{m}^{2}\right]: 1000 \times 2000 \text {. } \\
{ }^{*} \text { linear interpolated. }\end{array}$} \\
\hline
\end{tabular}



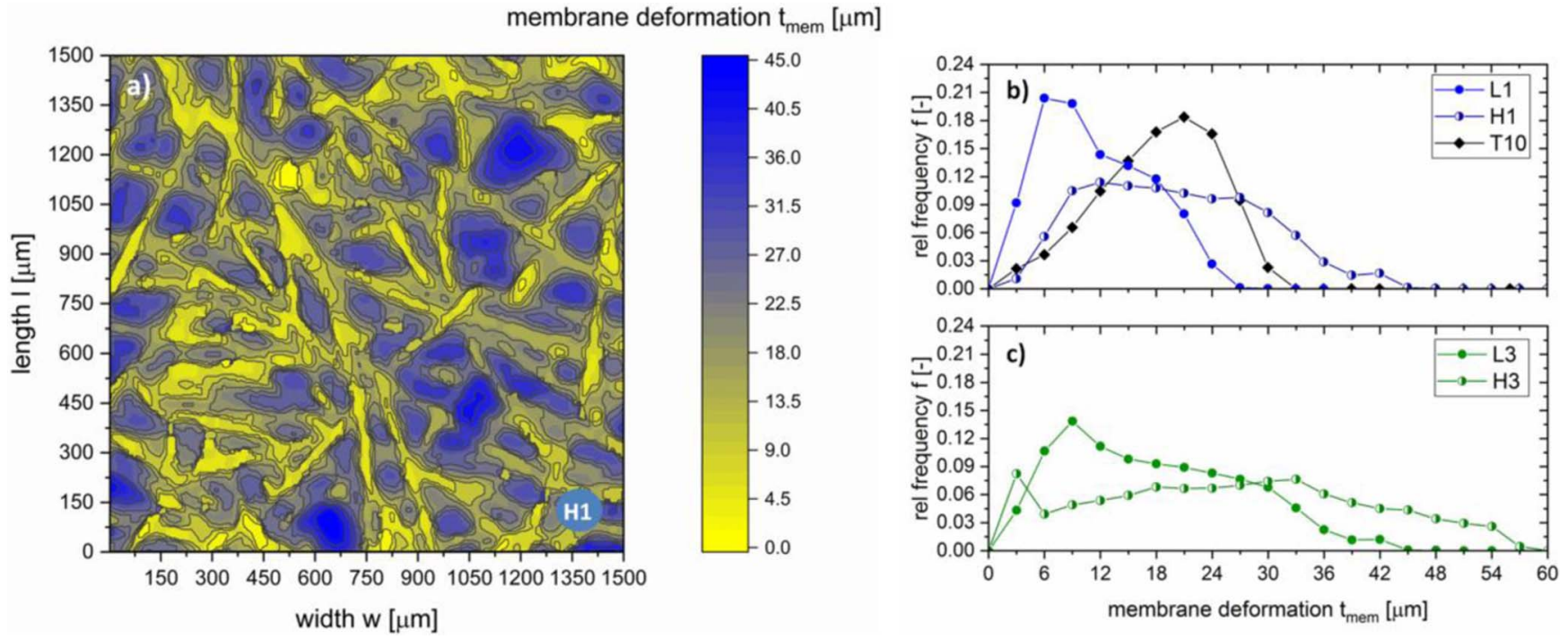

Figure 9. Membrane deformation analysis: a) color intensity scale map for membrane after operation with material H1, b) histograms for membrane deformation for after operation with materials H1, L1 and T10 and c) histograms for membrane deformation for materials L3 and H3.

fraction of $26 \%$ of the cumulative PTL surface height is covered. The results indicate that $\mathrm{H} 3$ with big solid sizes, high porosity, and large pores reveals the intrinsic deformation limits of the membrane.

Membrane deformation.-The deformation of the catalyst coated membrane defines its mechanical and electrochemical interaction with the PTL. Catalyst layers, as well as the membrane are plastically deformed by Ti fibers of the PTL. Figure 9a shows the imprint of the PTL H1 into the membrane after operation. The silhouettes of the fiber features are observed within the first $15 \mu \mathrm{m}$ in depth-direction due to membrane deformation. The membrane is deformed also by the second fiber layer before penetrating less accessible pores in deeper void domains. L1, L3, H1, H3 as well as T10 show similar trends as $\mathrm{H} 1$, their membrane deformation maps are given in the Supplementary Figure 3.

In Figure $9 \mathrm{~b}$ the catalyst/membrane deformation for $\mathrm{L} 1$ and $\mathrm{H} 1$ are plotted in a histogram, together with data for T10. Figure 9c shows the results of L3 and H3. L1 features the lowest mean membrane deformation $R_{m}^{\text {mem }}$ of $11.7 \mu \mathrm{m}$ due to the finest solid features and smallest pore sizes. Comparing to $\mathrm{H} 1$, it is observed that a change in porosity from $56 \%$ to $76 \%$ for identical fiber diameter results in a $30 \%$ increase of $R_{m}^{m e m}$ to $18.8 \mu \mathrm{m}$. The higher porosity and the related larger pore sizes of the $\mathrm{H}$ type samples promote membrane creeping and lead to broader membrane deformation distributions. Despite bigger pore sizes T10 features a sharper, less broad distribution than L3, H1 and $\mathrm{H} 3$ but a similar roughness $R_{m}^{m e m}$ of $18.3 \mu \mathrm{m}$. The complete deformation analysis is summarized in Table II.

$\mathrm{H} 3$ configuration with its big pores and high porosity was chosen to reveal the limits of Nafion 117's structural membrane integrity. Contact depths up to $57 \mu \mathrm{m}$ were obtained, implying the membrane deformation is limited to $\sim 25 \%$ with respect to hydrated membrane thickness of $200 \mu \mathrm{m} .{ }^{43,44}$

Obviously not the entire CCM is in contact with the PTL surface. High membrane deformation doesn't contribute to interfacial contact area due to deformation of membrane in the pore space and respectively missing contact to the PTL surface. For the determination of the interfacial contact area (see next section) utilization of membrane deformation at $90 \%$ was identified to be most representative. The use of higher deformation fractions would result in an over-prediction of the contact area due to the asymptotic behavior of cumulative deformations above $90 \%$ and missing contact of PTL and CCM. The cumulative frequency of membrane deformation height is given in Supplementary Figure 4.
Surface area and interfacial contact area.-Based on the surface roughness map of the transport layer its specific surface area $R_{S A}(d)$ can be determined. $R_{S A}(d)$ for the different materials as function of PTL depth $d$ is shown in Figure 10.

The available contact area increases with increasing PTL depth. The overall trend shows larger $R_{S A}(d)$ for smaller fiber diameters at the same porosity. Whereas at same fiber diameters, an increase in porosity results in a significant decrease of available PTL surface area.

A valuable parameter for comparing the different materials is the equivalent depth $d_{e q}$ at which the contact area $R_{S A}(d)$ reaches unity of $1.0 \mathrm{~m}^{2} / \mathrm{m}_{\mathrm{geo}}{ }^{2} . d_{e q}$ represents the required minimum membrane deformation to equal the contact area of a flat Ti plate with a surface roughness of $0 \mu \mathrm{m}$. L1, L2 as well as T10 reach the equivalent surface of $1.0 \mathrm{~m}^{2} / \mathrm{m}_{\text {geo }}{ }^{2}$ within $9-11 \mu \mathrm{m}$. Bigger fiber diameters and higher porosities result in depths of $19-32 \mu \mathrm{m}(\mathrm{L} 3, \mathrm{H} 1$ and $\mathrm{H} 2)$, while

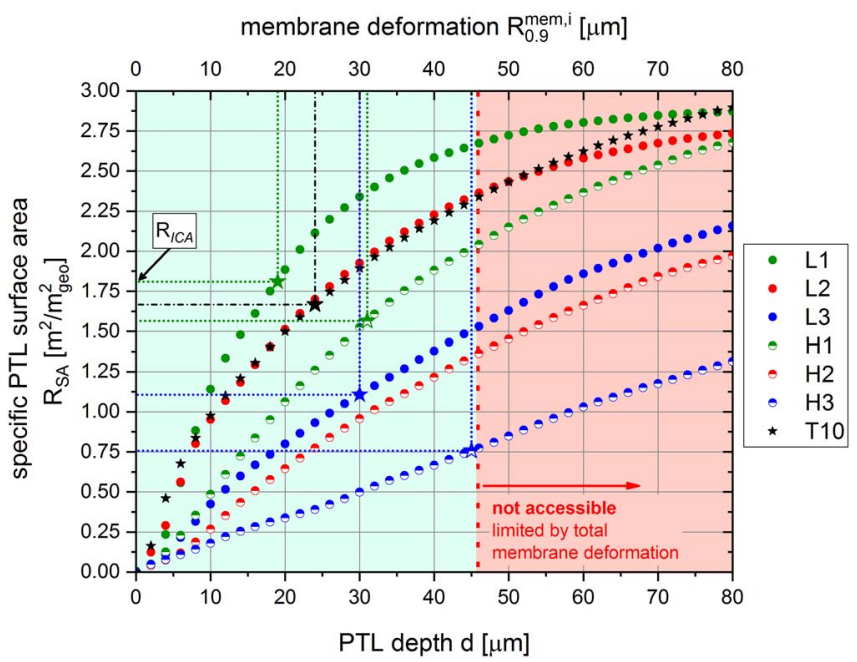

Figure 10. The specific surface area $R_{S A}$ as function of PTL depth $d$ is plotted for the fiber materials as well as for powder sintered PTL T10. Membrane deformations $R_{0.9}^{m e m, i}$, represented by asterisks, are included for different PTL types $i$. Intersections of the horizontal dashed lines with the $y$-axis reveals the specific interfacial contact areas $R_{I C A}$ of corresponding PTL/CL contact area under cell operation. The ICAs in the green domain are PTL structural dependent whereas red area highlights inaccessible contact surfaces due to limited maximum membrane deformation of membrane N117. 
for $\mathrm{H} 3 d_{e q}$ is even $58 \mu \mathrm{m}$. As thinner membranes of i.e. $50 \mu \mathrm{m}$ are used, membrane deformation is more limited than the experimental values in this study for $175 \mu \mathrm{m}$ (dry thickness of Nafion 117). The specific PTL surface area values are therefore also calculated for a depth of $10 \mu \mathrm{m}$ and are compared in Table II. The $R_{S A}(10 \mu \mathrm{m})$ values are between 0.2 and $1.1 \mathrm{~m}^{2} / \mathrm{m}_{\text {geo }}{ }^{2}$ indicating that the coarser PTL materials would not be suited for operation with thinner membranes as due to a lack of contact area and associated high local pressures at the interface between PTL and CL.

The actual interfacial contact areas under operation were calculated by combining the PTL based specific surface area and the experimentally determined membrane deformation according to Eq. 6. Values for the specific interfacial contact area were found to be $1.9>R_{I C A}>$ 0.7 . While the materials with thin fiber diameters (L1, L2 and $\mathrm{H} 1)$ have high specific interfacial contact areas in the range of $1.6-1.8 \mathrm{~m}^{2} / \mathrm{m}_{\text {geo }}{ }^{2}$, the coarse fiber materials L3 and H3 show significantly lower values of 1.1 and $0.76 \mathrm{~m}^{2} / \mathrm{m}_{\text {geo }}{ }^{2}$, respectively. T10 has a relatively high $R_{I C A}$ of $1.7 \mathrm{~m}^{2} / \mathrm{m}_{\text {geo }}{ }^{2}$ due to its low porosity.

The $R_{I C A}$ is limited by two effects, first by the maximum mechanical membrane deformation and second the PTL topology. The two regimes are classified by different color in Figure 10. For Nafion 117 , a maximum deformation of 40-50 $\mu \mathrm{m}$ is observed, so for depth values $<45 \mu \mathrm{m}$ the PTL structure determines the interfacial contact area (green area in Figure 10). For PTL depth $>45 \mu \mathrm{m}$ the contact area is limited by the intrinsic maximum membrane deformation. For thinner membranes the green area will be reduced to penetration depth $<45 \mu \mathrm{m}$, because of lower absolute membrane deformations. H3 has the most extreme properties for the interference between PTL and CCM with by far lowest specific ICA.

The low values in $R_{I C A}$ also imply a heterogeneous contact pressure distribution where the local pressure at the interface of PTL and CL scales with $R_{I C A}^{-1}$. This effect needs to be considered especially when screening PTLs in long-term and degradation tests. The combination of high membrane deformation and low ICA appears to represent a potential source for triggering mechanical membrane as well as catalyst layer degradation due to localized contact pressure and resulting stress and strain at the PTL/CL interface..$^{1,45}$

\section{Conclusions}

In this study, morphological properties of a systematically varied porous transport layer material matrix with 2 porosities ( $56 \%$ and $76 \%$ ) and three fiber diameters $(11 \mu \mathrm{m}, 15 \mu \mathrm{m}, 30 \mu \mathrm{m})$ were determined and compared to a conventional Titanium powder sintered material (T10 from GKN). X-ray tomographic microscopy analysis of the bulk properties was based on reliable ROI's up to 20 times larger than the representative equivalent volume for porosity. The pore size distribution analysis showed sharp solid and void spectra. Mercury intrusion porosimetry revealed limiting pore throat sizes that can function as possible bottlenecks for the gas/liquid transport during PEWE operation. Fibre based materials feature improved gas as well as liquid transport properties of up to 2 order in magnitude for permeability and 4 times higher diffusivity with respect to powder sinter material T10 whereas T10 outperforms fiber materials in thermal conductivity by $10 \mathrm{~W}(\mathrm{mK})^{-1}$ versus $2-6 \mathrm{~W}(\mathrm{mK})^{-1}$, driven by its significantly lower porosity. PTL Fiber orientations were correlated to computed transport parameters in through-plane and in-plane direction. A broad fiber distribution was identified to be beneficial for isotropic in-plane conductivities. No significant impact was found for gas and liquid transport parameter.

As surface properties of the porous transport layer materials may also play an important role for the electrochemical performance in PEWE, a special focus was put on the analysis of the 3D surface roughness. Surfaces revealed an empirical linear correlation of increasing roughness with growing mean fiber diameter. A new surface parameter of electrochemical interest, the specific interfacial contact area $\mathrm{R}_{\mathrm{ICA}}$ is introduced to quantify the interface between CL and PTL based on post operando membrane deformation data. For the fiber materials $\mathrm{R}_{\mathrm{ICA}}$ is 0.8 to $1.8 \mathrm{~m}^{2} / \mathrm{m}^{2}$ geo decreasing significantly with larger fiber di- ameters and higher porosities. GKN T10 has a value of $1.7 \mathrm{~m}^{2} / \mathrm{m}^{2}$ geo. The analysis of $R_{I C A}$ also implies a heterogeneous contact pressure distribution where the local pressure at the interface of PTL and CL scales with $R_{I C A}^{-1}$.

The plastic membrane deformation is a function of the PTL surface structure as well as structural membrane integrity. It was in the range of $19 \mu \mathrm{m}$ to $45 \mu \mathrm{m}$ for Nafion N117 membranes, however the specific interfacial contact area is limited by the maximum membrane deformation of about $45 \mu \mathrm{m}$. Use of thin membranes with $10 \mu \mathrm{m}$ deformation results in a decrease of $R_{I C A}$ up to a factor of 4 . This novel concept of ICA provides the possibility for quantitative assessment of the PTL surface. From the utilization of the catalyst layer its influence on the kinetic, ohmic and mass transport overpotentials can be judged and from the deformation of the membrane the influence on gas cross-over and (mechanical) membrane degradation assessed.

The influence of the structural bulk and surface properties analyzed in this work will be correlated to the electrochemical performance of these materials in PEWE in the second paper of this series. Based on this correlation, obtained for the systematic matrix of fiber materials, general governing parameters for porous transport layers for optimal PEWE performance will be identified and design rules deduced.

\section{Acknowledgments}

The authors thank the Swiss Federal Office of Energy (SFOE, grant no SI/501331-01) for funding of the project. TJS thanks Innosuisse and the SCCER Heat \& Electricity Storage. Jens Eller, Adrien Lamibrac and Thomas Gloor are gratefully acknowledged for their scientific and technical support.

\section{ORCID}

Thomas J. Schmidt (D) https://orcid.org/0000-0002-1636-367X

Felix N. Büchi (D) https://orcid.org/0000-0002-3541-4591

\section{References}

1. S. Stucki, G. G. Scherer, S. Schlagowski, and E. Fischer, Journal of Applied Electrochemistry, 28, 1041 (1998).

2. J. Rogelj, G. Luderer, R. C. Pietzcker, E. Kriegler, M. Schaeffer, V. Krey, and K. Riahi, Nature Climate Change, 5, 519 (2015).

3. M. Carmo, D. L. Fritz, J. Mergel, and D. Stolten, International Journal of Hydrogen Energy, 38, 4901 (2013).

4. O. Schmidt, A. Gambhir, I. Staffell, A. Hawkes, J. Nelson, and S. Few, International Journal of Hydrogen Energy, 42, 30470 (2017).

5. M. Suermann, K. Takanohashi, A. Lamibrac, T. J. Schmidt, and F. N. Büchi, Journal of The Electrochemical Society, 164, F973 (2017).

6. C. H. Lee, R. Banerjee, F. Arbabi, J. Hinebaugh, and A. Bazylak, V001T07A003 (2016).

7. U. Babic, M. Suermann, F. N. Büchi, L. Gubler, and T. J. Schmidt, Journal of The Electrochemical Society, 164, F387 (2017).

8. Z. Kang, J. Mo, G. Yang, S. Retterer, D. Cullen, T. Toops, J. Green Jr, M. Mench, and F.-Y. Zhang, Energy and Environmental Science, 10, 166 (2017).

9. B. Nils, C. Carsten, P. Karsten, and T. Jens, Energy Technology, 4, 212 (2016).

10. S. Siracusano, A. Di Blasi, V. Baglio, G. Brunaccini, N. Briguglio, A. Stassi, R. Ornelas, E. Trifoni, V. Antonucci, and A. S. Aricò, International Journal of Hydrogen Energy, 36, 3333 (2011).

11. P. Lettenmeier, S. Kolb, F. Burggraf, A. S. Gago, and K. A. Friedrich, J. Power Sources, 311, 153 (2016)

12. S. A. Grigoriev, P. Millet, S. A. Volobuev, and V. N. Fateev, International Journal of Hydrogen Energy, 34, 4968 (2009).

13. A. Fallisch, L. Schellhase, J. Fresko, M. Zechmeister, M. Zedda, J. Ohlmann, L. Zielke, N. Paust, and T. Smolinka, International Journal of Hydrogen Energy, 42, 13544 (2017).

14. H. Ito, T. Maeda, A. Nakano, A. Kato, and T. Yoshida, Electrochim Acta, 100, 242 (2013).

15. H. Ito, T. Maeda, A. Nakano, C. M. Hwang, M. Ishida, A. Kato, and T. Yoshida, International Journal of Hydrogen Energy, 37, 7418 (2012).

16. I. Dedigama, P. Angeli, K. Ayers, J. B. Robinson, P. R. Shearing, D. Tsaoulidis, and D. J. L. Brett, International Journal of Hydrogen Energy, 39, 4468 (2014)

17. I. Dedigama, P. Angeli, N. van Dijk, J. Millichamp, D. Tsaoulidis, P. R. Shearing, and D. J. L. Brett, J. Power Sources, 265, 97 (2014)

18. F. Arbabi, A. Kalantarian, R. Abouatallah, R. Wang, J. S. Wallace, and A. Bazylak, J. Power Sources, 258, 142 (2014) 
19. F. Arbabi, H. Montazeri, R. Abouatallah, R. Wang, and A. Bazylak, Journal of The Electrochemical Society, 163, F3062 (2016).

20. J. Seweryn, J. Biesdorf, T. J. Schmidt, and P. Boillat, Journal of The Electrochemical Society, 163, F3009 (2016).

21. O. Panchenko, E. Borgardt, W. Zwaygardt, F. J. Hackemüller, M. Bram, N. Kardjilov, T. Arlt, I. Manke, M. Müller, D. Stolten, and W. Lehnert, J Power Sources, 390, 108 (2018)

22. M. A. Hoeh, T. Arlt, I. Manke, J. Banhart, D. L. Fritz, W. Maier, and W. Lehnert, Electrochemistry Communications, 55, 55 (2015).

23. E. Leonard, A. D. Shum, S. Normile, D. C. Sabarirajan, D. G. Yared, X. Xiao, and I. V. Zenyuk, Electrochim Acta, 276, 424 (2018).

24. L. Zielke, A. Fallisch, N. Paust, R. Zengerle, and S. Thiele, Rsc Adv, 4, 58888 (2014)

25. J. Mo, Z. Kang, G. Yang, Y. Li, S. T. Retterer, D. A. Cullen, T. J. Toops, G. Bender, B. S. Pivovar, J. B. Green Jr, and F.-Y. Zhang, J Mater Chem A, 5, 18469 (2017)

26. C.-M. Hwang, M. Ishida, H. Ito, T. Maeda, A. Nakano, A. Kato, and T. Yoshida, Journal of International Council on Electrical Engineering, 2, 171 (2012).

27. C. M. Hwang, M. Ishida, H. Ito, T. Maeda, A. Nakano, A. Kato, and T. Yoshida, Power Sources, 202, 108 (2012).

28. P. Lettenmeier, S. Kolb, N. Sata, A. Fallisch, L. Zielke, S. Thiele, A. S. Gago, and K. A. Friedrich, Energy \& Environmental Science, 10, 2521 (2017).

29. M. Beat and H. Lorenz, Journal of the American Ceramic Society, 91, 4059 (2008).

30. J. H. Hubbell and S. M. Seltzer, X-Ray Mass Attenuation Coefficients, in, Radiation Physics Division PML NIST, https://www.nist.gov/pml/x-ray-massattenuation-coefficients.
31. N. Otsu, IEEE Transactions on Systems, Man, and Cybernetics, 9, 62 (1979).

32. T. Hildebrand and P. Rüegsegger, Journal of Microscopy, 185, 67 (1997)

33. Z. Püspöki, M. Storath, D. Sage, and M. Unser, in Focus on Bio-Image Informatics, W. H. De Vos, S. Munck, and J.-P. Timmermans Editors, p. 69, Springer International Publishing, Cham (2016).

34. M. Suermann, A. Pătru, T. J. Schmidt, and F. N. Büchi, International Journal of Hydrogen Energy, 42, 12076 (2017)

35. W. P. Dong, P. J. Sullivan, and K. J. Stout, Wear, 178, 45 (1994).

36. W. P. Dong, P. J. Sullivan, and K. J. Stout, Wear, 178, 29 (1994).

37. A. Lamibrac, J. Roth, M. Toulec, F. Marone, M. Stampanoni, and F. N. Büchi, Journal of The Electrochemical Society, 163, F202 (2016).

38. K. P. Shrestha and J. T. Gostick, in ECS Transactions, 50, 469 (2012).

39. J. Garcia-Navarro, M. Schulze, and K. A. Friedrich, ACS Sustainable Chemistry \& Engineering, 7, 1600 (2019)

40. C. Immerz, M. Schweins, P. Trinke, B. Bensmann, M. Paidar, T. Bystroň, K. Bouzek, and R. Hanke-Rauschenbach, Electrochim Acta, 260, 582 (2018).

41. N. Epstein, Chemical Engineering Science, 44, 777 (1989).

42. J. Mo, Z. Kang, S. T. Retterer, D. A. Cullen, T. J. Toops, J. B. Green, M. M. Mench, and F.-Y. Zhang, Science Advances, 2 (2016).

43. F. N. Büchi, B. Gupta, O. Haas, and G. G. Scherer, Electrochim Acta, 40, 345 (1995).

44. G. Gebel, P. Aldebert, and M. Pineri, Macromolecules, 20, 1425 (1987)

45. A. Laconti, H. Liu, C. Mittelsteadt, and R. McDonald, ECS Transactions, 1, 199 (2006). 